\title{
Neutral Pyridyl-Functionalized C,N-ortho-Chelated Aminoaryl Platinum(II) Corner Building Blocks for Application in Coordination Reactions
}

\author{
Catelijne H. M. Amijs, ${ }^{\dagger}$ Alexsandro Berger, ${ }^{\dagger}$ Fouad Soulimani, ${ }^{\ddagger}$ Tom Visser, ${ }^{\ddagger}$ Gerard P. M. van Klink, ${ }^{\dagger}$ \\ Martin Lutz,, Anthony L. Spek, ${ }^{*, \S}$ and Gerard van Koten ${ }^{*,+}$ \\ Organic Chemistry and Catalysis, Debye Institute, Utrecht University, Padualaan 8, \\ 3584 CH Utrecht, The Netherlands, Inorganic Chemistry and Catalysis, Debye Institute, Utrecht \\ University, Sorbonnelaan 16, 3584 CA Utrecht, The Netherlands, and Crystal and Structural \\ Chemistry, Bijvoet Center for Biomolecular Research, Utrecht University, Padualaan 8, \\ $3584 \mathrm{CH}$ Utrecht, The Netherlands
}

Received May 4, 2005

Two homoleptic pyridyl-functionalized $\mathrm{C,N}$-ortho-chelating aminoaryl platinum(II) complexes, cis-[Pt $\left.\left(\eta^{2}-\mathrm{C}, \mathrm{N}\right)\right](3 \mathbf{a}, \mathbf{b})$, were prepared via an unconventional method involving the initial synthesis of a bromide-functionalized $C, N$-chelating aminoaryl platinum(II) precursor complex $\mathbf{8}$, to which subsequently pyridyl groups were attached via a SuzukiMiyaura $\mathrm{C}-\mathrm{C}$ coupling reaction. The electron-donating properties of the pyridyl nitrogen atoms of the resulting complexes $(3 \mathbf{a}, \mathbf{b})$ were used in complexation reactions with monocationic NCN-pincer $\left(\mathrm{NCN}=\left[\mathrm{C}_{6} \mathrm{H}_{3}\left(\mathrm{CH}_{2} \mathrm{NMe}_{2}\right)_{2}\right.\right.$ $2,6]^{-}$) platinum(II) (11a) and palladium(II) (12a) nitrate complexes [M(NCN)( $\left.\left(\mathrm{NO}_{3}\right)\right]$, thereby obtaining four trimetallic coordination complexes 16-19. The difference in the pyridine-metal coordination behavior between platinum and palladium was studied by varying the ratios of the reagents and by variable-temperature NMR experiments. IR and Raman analyses of 11a and 12a were performed to determine the coordination behavior of the nitrate counteranion, and it was found that both $\mathrm{NO}_{3}{ }^{-}$and $\mathrm{H}_{2} \mathrm{O}$ coordinate to the metal centers. The crystal structure determinations of free pyridyl complex 3a, $\left[\mathrm{Pt}(\mathrm{NCN})\left(\mathrm{NO}_{3}\right)\right](11 \mathrm{a})$, and $\left[\mathrm{Pt}(\mathrm{NCN})\left(\mathrm{NO}_{3}\right)\right] \cdot\left(\mathrm{H}_{2} \mathrm{O}\right)(11 \mathbf{b})$, as well as the crystal structure of trisplatinum coordination complex 16 , are reported.

\section{Introduction}

Since the first report of a self-assembled metallasupramolecular square, ${ }^{1}$ the synthesis of self-assembled molecular squares, cycles, and cages has received considerable attention $^{2}$ not only because of their novel structural features but also for their potential applications in the field of host-guest chemistry, catalysis, and luminescent materials and for molecular recognition and sensing. ${ }^{3}$ The two most important factors for the rational design of these coordination-based supramolecular entities are the symmetry and shape of the

\footnotetext{
* To whom correspondence should be addressed. E-mail: g.vankoten@ chem.uu.nl (G.v.K.; phone +31-30-2533120; fax +31-30-2523615); a.1.spek@chem.uu.nl (A.L.S. for crystallographic data only; phone +3130-2532538; fax +31-30-2523940).

Organic Chemistry and Catalysis, Debye Institute.

+ Inorganic Chemistry and Catalysis, Debye Institute.

$\S$ Crystal and Structural Chemistry, Bijvoet Center for Biomolecular Research.

(1) Fujita, M.; Yazaki, J.; Ogura, K. J. Am. Chem. Soc. 1990, 112, 56455647.

(2) (a) Seidel, S. R.; Stang, P. J. Acc. Chem. Res. 2002, 35, 972-983. (b) Fujita, M.; Umemoto, K.; Yoshizawa, M.; Fujita, N.; Kusukawa, T.; Biradha, K. Chem. Commun. 2001, 509-518.
}

10.1021/ic050697v CCC: $\$ 30.25$ C 2005 American Chemical Society Published on Web 08/16/2005 resulting assembly. These two factors are determined solely by the type and properties of the building blocks. For twodimensional structures, two types of building blocks are required: (1) linear ditopic units, which contain reactive sites with a $180^{\circ}$ orientation relative to each other, and (2) angular ditopic units, possessing reactive sites with other desirable fixed angles. Generally, a building block consists of electrondeficient metal centers or organic ligands with Lewis base donor functionalities (often pyridine). Square-planar platinum(II) and palladium(II) centers with coordination angles of $180^{\circ}$ or $90^{\circ}$ are frequently used as either linear or corner acceptor units, respectively. Only in a few cases have organometallic complexes been used as donor building blocks. In these cases, the metal center itself is not used as the acceptor unit but only as a center for determining the orientation of the donor units via its attached ligands. The advantages of such complexes are their predefined structures,

(3) For a recent review on the synthesis and application of metallomolecular squares, see: (a) Würthner, F.; You, C.-C.; Saha-Möller, C. R. Chem. Soc. Rev. 2004, 33, 133-146. (b) Amijs, C. H. M.; van Klink, G. P. M.; van Koten, G. Dalton Trans., in press.

Inorganic Chemistry, Vol. 44, No. 19, 2005 
Amijs et al.

Chart 1. Organometallic Corner Complexes

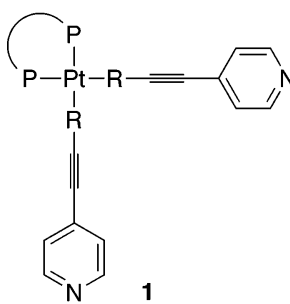

$\mathrm{R}=$ none, $\mathrm{C}_{6} \mathrm{H}_{4}$

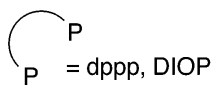

e.g., in the case of square-planar complexes linear or angular, and their high chemical and physical stability compared to coordination complexes. An additional feature is that especially the $90^{\circ}$ angle is rarely observed in pure organic donor compounds. Examples of right-angled electron-donating structures are complexes $\mathbf{1}$ and $\mathbf{2}$ (Chart 1) reported by Stang and Ferrer. ${ }^{4}$

For our research, we aimed at the use of the predefined $90^{\circ}$ angle of organometallic cis-platinum(II) complexes as molecular building blocks representing a right-angled corner. The homoleptic platinum(II) complex [Pt(dmba $\left.)_{2}\right](\mathrm{dmba}=$ $\left.\left[\mathrm{C}_{6} \mathrm{H}_{4}\left\{\mathrm{CH}_{2} \mathrm{NMe}_{2}\right\}-2\right]^{-}\right)^{5}$ was chosen as the corner building block. On the basis of this complex, we reported in a previous paper the synthesis of functionalized cis- and trans- $\mathrm{C}, \mathrm{N}$ ortho-chelated aminoaryl platinum(II) compounds. ${ }^{6}$ The functionalities on the aryl groups (halides or methyl-, naphthyl-, or (dimethylamino)methyl groups) were used to influence the cis/trans ratio and for additional functionalization of these complexes by means of lithiation, transmetalation, and $\mathrm{C}-\mathrm{C}$ coupling reactions. We describe here the synthesis of pyridyl-functionalized cis- $\mathrm{C}, \mathrm{N}$-ortho-chelated aminoaryl platinum(II) corner complexes (3a,b, Chart 1) and their use as molecular building blocks with donor functionalities in self-assembly reactions with electron-deficient metal centers.

A common route for the synthesis of organometallic complexes comprises the construction of the organic backbone, followed by the introduction of the metal center in the final step. However, for successful synthesis of complexes 3a and 3b, we had to apply an unconventional synthesis route, which implies the use of metal-catalyzed $\mathrm{C}-\mathrm{C}$ coupling reactions of pyridyl reagents with organoplatinum precursor complexes. The reactivity of these donor complexes in assembly reactions was tested with monocationic platinum(II) and palladium(II) NCN-pincer nitrate complexes $\left(\mathrm{NCN}=\left[\mathrm{C}_{6} \mathrm{H}_{3}\left(\mathrm{CH}_{2} \mathrm{NMe}_{2}\right)_{2}-2,6\right]^{-}\right)$. For the latter complexes, the coordination behavior of the nitrate counteranion was investigated.

(4) (a) Manna, J.; Whiteford, J. A.; Stang, P. J. J. Am. Chem. Soc. 1996, 118, 8731-8732. (b) Müller, C. M.; Whiteford, J. A.; Stang, P. J. J. Am. Chem. Soc. 1998, 120, 9827-9837. (c) Stang, P. J. Chem. Eur. J. 1998, 4, 19-27. (d) Ferrer, M.; Rodríguez, L.; Rossell, O.; Solans, X. J. Organomet. Chem. 2005, 690, 1612-1619.

(5) Longoni, G.; Fantucci, P.; Chini, P.; Canziani, F. J. Organomet. Chem. 1972, 39, 413-425.

(6) Amijs, C. H. M.; van Klink, G. P. M.; Lutz, M.; Spek, A. L.; van Koten, G. Organometallics 2005, 24, 2944-2958.

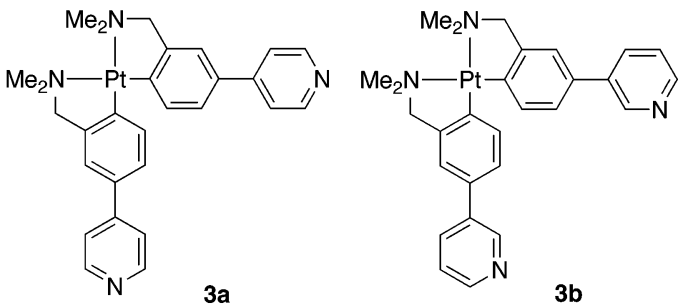

\section{Experimental Section}

General Procedures. 1-Bromo-2-(bromomethyl)-4-iodobenzene (4), ${ }^{7}$ 4-bromo-2-[(dimethylamino)methyl]iodobenzene (9), ${ }^{6}$ 4-pyridylborane pinacol ester, ${ }^{8}$ 3-pyridylborane pinacol ester, ${ }^{9}[\mathrm{Pt}(\mathrm{NCN})$ $\left.\left(\mathrm{H}_{2} \mathrm{O}\right)\right]\left(\mathrm{BF}_{4}\right)(\mathbf{1 1 c}),{ }^{10}\left[\mathrm{Pd}(\mathrm{NCN})\left(\mathrm{H}_{2} \mathrm{O}\right)\right]\left(\mathrm{BF}_{4}\right)(\mathbf{1 2 c}),{ }^{10}[\mathrm{PtCl}(\mathrm{NCN})]$ $(\mathbf{1 3}),{ }^{10}$ and $[\mathrm{PdCl}(\mathrm{NCN})](\mathbf{1 4})^{11}$ were synthesized according to (modified) literature procedures. All solvents and other reagents were obtained from commercial sources and were used without further purification unless stated otherwise. Reactions involving organolithium derivatives were carried out under an inert atmosphere of dry, oxygen-free nitrogen using standard Schlenk techniques. $\mathrm{Et}_{2} \mathrm{O}$ and THF were distilled from $\mathrm{Na} /$ benzophenone prior to use. ${ }^{1} \mathrm{H},{ }^{13} \mathrm{C}$, and ${ }^{195} \mathrm{Pt}$ NMR spectra were recorded at 300 , 75.5, and 64.3 $\mathrm{MHz}$, respectively, at $25^{\circ} \mathrm{C}$. Chemical shifts are reported in ppm. IR spectra were recorded on a Perkin-Elmer Spectrum One FT-IR spectrometer. Raman spectra of solids were measured on a Perkin-Elmer 2000 FT-Raman spectrometer (excitation wavelength $1064 \mathrm{~nm}$ ) and those of aqueous solutions on a Kaiser RXN 532-nm dispersive instrument. The applied optical resolution was $4 \mathrm{~cm}^{-1}$. Elemental analyses were performed by Kolbe, Mikroanalytisches Laboratorium (Mülheim a.d. Ruhr, Germany).

1-Bromo-2-[(dimethylamino)methyl]-4-iodobenzene (5). To a solution of 4 (17.1 g, $45.5 \mathrm{mmol})$ was added dimethylamine (60 $\mathrm{mL}, 865 \mathrm{mmol}, 19$ equiv) in $\mathrm{Et}_{2} \mathrm{O}(250 \mathrm{~mL})$ at room temperature. After $2 \mathrm{~h}$, the salts were removed by filtration and the reaction mixture was concentrated in vacuo. The resulting oil was purified by flash distillation. Yield: $12.6 \mathrm{~g}(37.0 \mathrm{mmol}, 81 \%) .{ }^{1} \mathrm{H}$ NMR $\left(\mathrm{CDCl}_{3}\right): \delta 2.30\left(\mathrm{~s}, 6 \mathrm{H}, \mathrm{NCH}_{3}\right), 3.46\left(\mathrm{~s}, 2 \mathrm{H}, \mathrm{CH}_{2} \mathrm{~N}\right), 7.26\left(\mathrm{~d},{ }^{3} \mathrm{~J}_{\mathrm{H}, \mathrm{H}}\right.$ $=8 \mathrm{~Hz}, 1 \mathrm{H}, \operatorname{ArH}-6), 7.42\left(\mathrm{~d},{ }^{3} J_{\mathrm{H}, \mathrm{H}}=10 \mathrm{~Hz}, 1 \mathrm{H}, \operatorname{ArH}-5\right), 7.78(\mathrm{~s}$, $1 \mathrm{H}, \mathrm{ArH}-3) .{ }^{13} \mathrm{C}\left\{{ }^{1} \mathrm{H}\right\} \mathrm{NMR}\left(\mathrm{CDCl}_{3}\right): \delta 45.7\left(\mathrm{NCH}_{3}\right), 62.9\left(\mathrm{CH}_{2} \mathrm{~N}\right)$, 92.8 (ArC-4), 124.3 (ArC-1), 134.4, 137.4, 139.5, 140.7 (ArC-3). Anal. Calcd for $\mathrm{C}_{9} \mathrm{H}_{11} \mathrm{NBrI}$ : C, 31.79; H, 3.26; N, 4.12. Found: C, 31.87; H, 3.35; N, 4.18.

1-Bromo-2-[(dimethylamino)methyl]-4-( $p$-pyridyl)benzene (6). Compound 5 (1.39 g, $4.09 \mathrm{mmol})$, 4-pyridylborane pinacol ester (0.88 g, 4.29 mmol, 1.05 equiv), [ $\left.\mathrm{PdCl}_{2}(\mathrm{dppf})\right]$ (0.4 mmol, $10 \mathrm{~mol}$

(7) Amijs, C. H. M.; van Klink, G. P. M.; van Koten, G. Green Chem 2003, 5, 470-474.

(8) Coudret, C. Synth. Commun. 1996, 26, 3543-3547.

(9) Li, W.; Nelson, D. P.; Jensen, M. S.; Hoerrner, R. S.; Cai, D.; Larsen, R. D.; Reider, P. J. J. Org. Chem. 2002, 67, 5394-5397.

(10) (a) Chuchuryukin, A. V.; Dijkstra, H. P.; Suijkerbuijk, B. M. J. M.; Klein Gebbink, R. J. M.; van Klink, G. P. M.; Mills, A. M.; Spek, A L.; van Koten, G. Angew. Chem., Int. Ed. 2003, 42, 228-230. (b) Chuchuryukin, A. V.; Dijkstra, H. P.; Chase, P. A.; Suijkerbuijk, B. M. J. M.; Mills, A. M.; Spek, A. L.; van Klink, G. P. M.; van Koten, G. Adv. Synth. Catal. 2005, 347, 447-462.

(11) Steenwinkel, P.; Gossage, R. A.; Maunula, T.; Grove, D. M.; van Koten, G. Chem. Eur. J. 1998, 4, 763-768. 
$\%$ ), and $\mathrm{Na}_{2} \mathrm{CO}_{3}$ (2.6 g, 24.5 mmol, 6 equiv) were brought into a Schlenk flask and evacuated for $30 \mathrm{~min}$. To this was added a degassed mixture of DME $(60 \mathrm{~mL})$ and $\mathrm{H}_{2} \mathrm{O}(30 \mathrm{~mL})$. The reaction mixture was stirred at $90{ }^{\circ} \mathrm{C}$ for $40 \mathrm{~h}$. The reaction mixture was cooled to room temperature, extracted with $\mathrm{Et}_{2} \mathrm{O}(50 \mathrm{~mL})$, dried over $\mathrm{MgSO}_{4}$, filtered, and evaporated to dryness. The product was purified by column chromatography (silica, 94:3:3 $\mathrm{CH}_{2} \mathrm{Cl}_{2} / \mathrm{NEt}_{3} /$ $\mathrm{MeOH})$. Compound 6 was isolated as a light yellow oil. Yield: $0.69 \mathrm{~g}(2.37 \mathrm{mmol}, 58 \%) .{ }^{1} \mathrm{H} \mathrm{NMR}\left(\mathrm{CDCl}_{3}\right): \delta 2.33\left(\mathrm{~s}, 6 \mathrm{H}, \mathrm{NCH}_{3}\right)$, $3.58\left(\mathrm{~s}, 2 \mathrm{H}, \mathrm{CH}_{2} \mathrm{~N}\right), 7.37\left(\mathrm{~d},{ }^{3} J_{\mathrm{H}, \mathrm{H}}=8 \mathrm{~Hz}, 1 \mathrm{H}, \mathrm{ArH}-6\right), 7.50(\mathrm{dd}$, $\left.{ }^{3} J_{\mathrm{H}, \mathrm{H}}=6 \mathrm{~Hz},{ }^{2} J_{\mathrm{H}, \mathrm{H}}=2 \mathrm{~Hz}, 2 \mathrm{H}, \mathrm{ArH}-8,11\right), 7.65\left(\mathrm{~d},{ }^{3} J_{\mathrm{H}, \mathrm{H}}=8 \mathrm{~Hz}\right.$, $1 \mathrm{H}, \operatorname{ArH}-5), 7.73(\mathrm{~s}, 1 \mathrm{H}, \mathrm{ArH}-3), 8.64\left(\mathrm{dd},{ }^{3} J_{\mathrm{H}, \mathrm{H}}=6 \mathrm{~Hz},{ }^{2} J_{\mathrm{H}, \mathrm{H}}=\right.$ $2 \mathrm{~Hz}, 2 \mathrm{H}, \mathrm{ArH}-9,10) .{ }^{13} \mathrm{C}\left\{{ }^{1} \mathrm{H}\right\} \mathrm{NMR}\left(\mathrm{CDCl}_{3}\right): \delta 45.5\left(\mathrm{NCH}_{3}\right)$, $64.4\left(\mathrm{CH}_{2} \mathrm{~N}\right), 121.8,125.8,127.7,129.1,129.9,138.3,140.1,148.4$, 150.3. Anal. Calcd for $\mathrm{C}_{14} \mathrm{H}_{15} \mathrm{~N}_{2} \mathrm{Br}$ : C, 57.75; H, 5.19; N, 9.62. Found: C, 58.02; H, 5.31; N, 9.49.

cis-[Pt $\left.\left(\mathrm{C}_{6} \mathrm{H}_{3} \mathrm{Br}-4-\left\{\mathrm{CH}_{2} \mathrm{NMe}_{2}\right\}-2\right)_{2}\right](\mathbf{8})$. To a solution of 9 (2.11 $\mathrm{g}, 6.21 \mathrm{mmol})$ in $\mathrm{Et}_{2} \mathrm{O}(20 \mathrm{~mL})$ was added $n$-BuLi $(6.2 \mathrm{mmol}, 1.6$ $\mathrm{M}$ in hexane, $3.9 \mathrm{~mL}, 1$ equiv) at $-78{ }^{\circ} \mathrm{C}$. The reaction mixture was stirred for $5 \mathrm{~min}$, after which a suspension of cis-[ $\left.\mathrm{PtCl}_{2}(\mathrm{cod})\right]$ ( $1.11 \mathrm{~g}, 2.97 \mathrm{mmol}, 0.48$ equiv) in $\mathrm{Et}_{2} \mathrm{O}(10 \mathrm{~mL})$ was added. The off-white suspension was allowed to warm to room temperature and stirred for $3 \mathrm{~h}$. The precipitate was isolated by centrifugation and washed with water $(6 \mathrm{~mL}, 3 \times)$ and acetone $(6 \mathrm{~mL}, 2 \times)$ to give $1.70 \mathrm{~g}$ of $\mathbf{8}(2.74 \mathrm{mmol}, 92 \%)$ as a white solid. The analytical data were identical with the data previously reported. ${ }^{6}$

cis-[Pt(\{4-pyridyl $\}$-dmba $\left.)_{2}\right]$ (3a) and cis-[Pt(\{3-pyridyl $\}$ dmba $)_{2}$ ] (3b). Compound 8 (0.40 g, $\left.0.64 \mathrm{mmol}\right)$, 4- or 3-pyridylborane pinacol ester $\left(0.33 \mathrm{~g}, 1.61 \mathrm{mmol}, 2.5\right.$ equiv), $\left[\mathrm{PdCl}_{2}(\mathrm{dppf})\right]$ (33 mg, $0.044 \mathrm{mmol}, 7 \%$ ), and $\mathrm{Na}_{2} \mathrm{CO}_{3}(0.34 \mathrm{~g}, 3.24 \mathrm{mmol}, 5$ equiv) were brought into a Schlenk flask and evacuated for $30 \mathrm{~min}$. To this was added a degassed mixture of DME (15 mL), THF (15 $\mathrm{mL})$, and $\mathrm{H}_{2} \mathrm{O}(15 \mathrm{~mL})$. The reaction mixture was stirred at $70{ }^{\circ} \mathrm{C}$ until the reaction was complete $( \pm 3 \mathrm{~h})$. The reaction mixture was cooled to room temperature, extracted with $\mathrm{CH}_{2} \mathrm{Cl}_{2}(50 \mathrm{~mL})$, dried over $\mathrm{MgSO}_{4}$, filtered, and evaporated to dryness. The product was purified by column chromatography (basic alumina, from pure $\mathrm{CH}_{2}$ $\mathrm{Cl}_{2}$ to a mixture of 95:5 $\mathrm{CH}_{2} \mathrm{Cl}_{2} / \mathrm{NEt}_{3}$ ) and subsequently washed with $\mathrm{Et}_{2} \mathrm{O}(5 \mathrm{~mL}, 2 \times)$. Complexes $\mathbf{3 a}$ and $\mathbf{3 b}$ were isolated as light yellow solids. 3a. Yield: $0.31 \mathrm{~g}(0.50 \mathrm{mmol}, 78 \%) .{ }^{1} \mathrm{H} \mathrm{NMR}\left(\mathrm{CD}_{2^{-}}\right.$ $\left.\mathrm{Cl}_{2}\right): \delta 2.86\left(\mathrm{~s},{ }^{3} J_{\mathrm{Pt}, \mathrm{H}}=11 \mathrm{~Hz}, 12 \mathrm{H}, \mathrm{NCH}_{3}\right), 3.99\left(\mathrm{~s},{ }^{3} J_{\mathrm{Pt}, \mathrm{H}}=16\right.$ $\left.\mathrm{Hz}, 4 \mathrm{H}, \mathrm{CH}_{2} \mathrm{~N}\right), 7.35\left(\mathrm{dd},{ }^{3} J_{\mathrm{H}, \mathrm{H}}=8 \mathrm{~Hz},{ }^{4} J_{\mathrm{H}, \mathrm{H}}=2 \mathrm{~Hz}, 2 \mathrm{H}, \mathrm{ArH}-5\right)$, 7.40 (s, 2H, ArH-3), 7.53 (d, ${ }^{3} J_{\mathrm{Pt}, \mathrm{H}}$ not observed, ${ }^{3} J_{\mathrm{H}, \mathrm{H}}=8 \mathrm{~Hz}, 2 \mathrm{H}$, ArH-6), $7.58\left(\mathrm{dd},{ }^{3} J_{\mathrm{H}, \mathrm{H}}=5 \mathrm{~Hz},{ }^{2} J_{\mathrm{H}, \mathrm{H}}=2 \mathrm{~Hz}, 4 \mathrm{H}\right.$, ArH-8,11), 8.59 $\left(\mathrm{dd},{ }^{3} J_{\mathrm{H}, \mathrm{H}}=5 \mathrm{~Hz}, J_{\mathrm{H}, \mathrm{H}}=1 \mathrm{~Hz}, 4 \mathrm{H}, \operatorname{ArH}-9,10\right),{ }^{13} \mathrm{C}\left\{{ }^{1} \mathrm{H}\right\} \mathrm{NMR}$ $\left(\mathrm{CDCl}_{3}\right): \delta 50.3\left(\mathrm{NCH}_{3}\right), 73.7\left({ }^{2} J_{\mathrm{Pt}, \mathrm{C}}=51 \mathrm{~Hz}, \mathrm{CH}_{2} \mathrm{~N}\right), 119.6\left({ }^{3} J_{\mathrm{Pt}, \mathrm{C}}\right.$ $=37 \mathrm{~Hz}$, ArC-3), $121.0(\mathrm{ArC}-8,11), 124.4\left({ }^{3} J_{\mathrm{Pt}, \mathrm{C}}=87 \mathrm{~Hz}\right.$, ArC5), 132.1 (ArC), $139.5\left({ }^{2} J_{\mathrm{Pt}, \mathrm{C}}=48 \mathrm{~Hz}, \mathrm{ArC}-6\right), 141.9\left(\mathrm{ArC}_{\mathrm{ipso}}\right)$, 148.6, 149.1 (ArC-2), 150.2 (ArC-9,10). ${ }^{195} \mathrm{Pt} \mathrm{NMR}\left(\mathrm{CD}_{2} \mathrm{Cl}_{2}\right)$ : $\delta$ -3367.0. Anal. Calcd for $\mathrm{C}_{28} \mathrm{H}_{30} \mathrm{~N}_{4} \mathrm{Pt}$ : C, 54.45; H, 4.90; N, 9.07. Found: C, 54.30; H, 4.98; N, 8.87. 3b. Yield: $0.37 \mathrm{~g} \mathrm{(0.59} \mathrm{mmol,}$ 92\%). ${ }^{1} \mathrm{H} \mathrm{NMR}\left(\mathrm{CD}_{2} \mathrm{Cl}_{2}\right): \delta 2.84\left(\mathrm{~s},{ }^{3} \mathrm{~J}_{\mathrm{Pt}, \mathrm{H}}\right.$ not observed, $12 \mathrm{H}$, $\left.\mathrm{NCH}_{3}\right), 3.96\left(\mathrm{~s},{ }^{3} J_{\mathrm{Pt}, \mathrm{H}}\right.$ not observed, $\left.4 \mathrm{H}, \mathrm{CH}_{2} \mathrm{~N}\right), 7.22-7.30(\mathrm{~m}$, $6 \mathrm{H}, \mathrm{ArH}-3,5,10), 7.57\left(\mathrm{~d},{ }^{3} J_{\mathrm{H}, \mathrm{H}}=8 \mathrm{~Hz},{ }^{3} J_{\mathrm{Pt}, \mathrm{H}}=68 \mathrm{~Hz}, 2 \mathrm{H}, \mathrm{ArH}-\right.$ 6), $7.87\left(\mathrm{~d},{ }^{3} J_{\mathrm{H}, \mathrm{H}}=8 \mathrm{~Hz}, 2 \mathrm{H}, \mathrm{ArH}-11\right), 8.48\left(\mathrm{~d},{ }^{3} J_{\mathrm{H}, \mathrm{H}}=4 \mathrm{~Hz}, 2 \mathrm{H}\right.$, ArH-9), 8.85 (s, 2H, ArH-8). ${ }^{13} \mathrm{C}\left\{{ }^{1} \mathrm{H}\right\}$ NMR $\left(\mathrm{CDCl}_{3}\right): \delta 50.3$ $\left(\mathrm{NCH}_{3}\right), 73.7\left(\mathrm{CH}_{2} \mathrm{~N}\right), 119.8(\mathrm{ArC}-3), 123.5(\mathrm{ArC}-11), 124.5(\mathrm{ArC}-$ 5), 131.8, 133.7 (Ar-10), 137.5, 139.4 (ArC-6), 139.9 (ArC $\mathrm{Apso}_{\text {ipo }}$, 147.5, 148.0, 148.5 (ArC-2). ${ }^{195} \mathrm{Pt} \mathrm{NMR}\left(\mathrm{CD}_{2} \mathrm{Cl}_{2}\right): \delta-3375.8$. Anal. Calcd for $\mathrm{C}_{28} \mathrm{H}_{30} \mathrm{~N}_{4} \mathrm{Pt}$ : C, 54.45; H, 4.90; N, 9.07. Found: C, 54.32; H, 4.85; N, 8.87.

$\left[\mathrm{Pt}(\mathrm{NCN})\left(\mathrm{NO}_{3}\right)\right](\mathbf{1 1 a})$ and $\left[\mathrm{Pd}(\mathbf{N C N})\left(\mathrm{NO}_{3}\right)\right](\mathbf{1 2 a})$. A solution of 13 or $14(0.75 \mathrm{mmol})$ and $\mathrm{AgNO}_{3}(134 \mathrm{mg}, 0.79 \mathrm{mmol}, 1.1$ equiv) in acetone $(15 \mathrm{~mL})$ was stirred for $16 \mathrm{~h}$ in the absence of light. The reaction mixture was filtered over Celite, concentrated in vacuo, and washed with $\mathrm{Et}_{2} \mathrm{O}(3 \mathrm{~mL})$. Note: these reactions were performed in technical solvents, in open air. Complexes 11a and 12a were isolated as white solids. 11a. Yield: $0.32 \mathrm{~g}(0.71$ mmol, 95\%). The analytical data were identical with the data previously reported. ${ }^{12}{ }^{195} \mathrm{Pt}$ NMR (acetone- $\left.d_{6}\right): \delta-3299$. IR $\left(\mathrm{cm}^{-1}\right.$, solid): $1417_{\mathrm{s}} v_{1}\left(\mathrm{~A}^{\prime}\right), 1281_{\mathrm{vs}} v_{2}\left(\mathrm{~A}^{\prime \prime}\right), 1014_{\mathrm{m}} v_{3}\left(\mathrm{~A}^{\prime}\right), 839_{\mathrm{m}} v_{6}\left(\mathrm{~A}^{\prime \prime}\right)$, $768_{\mathrm{m}} v_{4}\left(\mathrm{~A}^{\prime}\right)$ or $v_{5}\left(\mathrm{~A}^{\prime}\right)\left(\mathrm{NO}_{3}^{-}\right)$. Raman $\left(\mathrm{cm}^{-1}\right): 1039_{\mathrm{s}} v_{1}\left(\mathrm{~A}_{1}^{\prime}\right), 1014_{\mathrm{s}}$ $v_{3}\left(\mathrm{~A}^{\prime}\right)$ (solid); $1046_{\mathrm{vs}} v_{1}\left(\mathrm{~A}_{1}^{\prime}\right)$ (aqueous solution). 11a $\cdot \mathbf{H}_{2} \mathbf{O}$. IR $\left(\mathrm{cm}^{-1}\right): 3573_{\mathrm{s}} v_{3}, 3494_{\mathrm{m}} v_{1}, 1613_{\mathrm{w}} v_{2}\left(\mathrm{H}_{2} \mathrm{O}\right) ; 1417_{\mathrm{m}} v_{1}\left(\mathrm{~A}^{\prime}\right), 1286_{\mathrm{vs}}$ $v_{2}\left(\mathrm{~A}^{\prime}\right), 1008_{\mathrm{m}} v_{3}\left(\mathrm{~A}^{\prime}\right), 838_{\mathrm{m}} v_{6}\left(\mathrm{~A}^{\prime \prime}\right), 769_{\mathrm{m}} v_{4}\left(\mathrm{~A}^{\prime}\right)$ or $v_{5}\left(\mathrm{~A}^{\prime}\right)\left(\mathrm{NO}_{3}{ }^{-}\right)$. Raman $\left(\mathrm{cm}^{-1}\right)$ : $1040_{\mathrm{s}} v_{1}\left(\mathrm{~A}_{1}^{\prime}\right), 1013_{\mathrm{s}} v_{3}\left(\mathrm{~A}^{\prime}\right) ; 1046_{\mathrm{vs}} v_{1}\left(\mathrm{~A}_{1}^{\prime}\right)$ (aqueous solution). 12a. Yield: $0.24 \mathrm{~g}(0.68 \mathrm{mmol}, 91 \%) .{ }^{1} \mathrm{H}$ NMR (acetone$\left.d_{6}\right): \delta 2.81\left(\mathrm{~s}, 12 \mathrm{H}, \mathrm{NCH}_{3}\right), 4.09\left(\mathrm{~s}, 4 \mathrm{H}, \mathrm{CH}_{2} \mathrm{~N}\right), 6.82\left(\mathrm{~d},{ }^{3} J_{\mathrm{H}, \mathrm{H}}=\right.$ $8 \mathrm{~Hz}, 2 \mathrm{H}, \mathrm{ArH}-3,5), 6.98\left(\mathrm{t},{ }^{3} J_{\mathrm{H}, \mathrm{H}}=7 \mathrm{~Hz}, 1 \mathrm{H}, \mathrm{ArH}-4\right) .{ }^{13} \mathrm{C}\left\{{ }^{1} \mathrm{H}\right\}$ NMR (acetone- $\left.d_{6}\right): \delta 52.1\left(\mathrm{NCH}_{3}\right), 74.2\left(\mathrm{CH}_{2} \mathrm{~N}\right), 120.8(\mathrm{ArC}-3,5)$, 125.6 (ArC-4), 146.4 (ArC-2,6), $153.1\left(\mathrm{C}_{\mathrm{ipso}}\right)$. IR ( $\mathrm{cm}^{-1}$, solid): $1411_{\mathrm{m}} v_{1}\left(\mathrm{~A}^{\prime}\right), 1289_{\mathrm{vs}} v_{2}\left(\mathrm{~A}^{\prime}\right), 1027_{\mathrm{m}} v_{3}\left(\mathrm{~A}^{\prime}\right), 840_{\mathrm{m}} v_{6}\left(\mathrm{~A}^{\prime \prime}\right), 764_{\mathrm{m}} v_{4}\left(\mathrm{~A}^{\prime}\right)$ or $v_{5}\left(\mathrm{~A}^{\prime}\right)\left(\mathrm{NO}_{3}{ }^{-}\right)$. Raman $\left(\mathrm{cm}^{-1}\right)$ : $1038_{\mathrm{s}} v_{1}\left(\mathrm{~A}_{1}^{\prime}\right), 1025_{\mathrm{s}} v_{3}\left(\mathrm{~A}^{\prime}\right)$ (solid); $1047_{\mathrm{vs}} v_{1}\left(\mathrm{~A}_{1}^{\prime}\right)$ (aqueous solution). Anal. Calcd for $\mathrm{C}_{12} \mathrm{H}_{19} \mathrm{~N}_{3} \mathrm{O}_{3}-$ Pd: C, 40.07; H, 5.32; N, 11.68. Found: C, 39.92; H, 5.26; N, 11.59.

Attempted Synthesis of $\left[\mathrm{Pt}(\mathrm{NCN})\left(\mathrm{H}_{2} \mathrm{O}\right)\right]\left(\mathrm{NO}_{3}\right)(11 \mathrm{~b})$ and [Pd$\left.(\mathbf{N C N})\left(\mathrm{H}_{2} \mathrm{O}\right)\right]\left(\mathbf{N O}_{3}\right)(\mathbf{1 2 b})$. A solution of 11a or 12a in $\mathrm{H}_{2} \mathrm{O}$ was stirred at room temperature for $16 \mathrm{~h}$, after which the solvent was evaporated by means of freeze-drying. The products were isolated as white solids. 11b. ${ }^{195} \mathrm{Pt} \mathrm{NMR}\left(\mathrm{D}_{2} \mathrm{O}\right): \delta-3351$. IR $\left(\mathrm{cm}^{-1}\right.$, solid): $3572_{\mathrm{w}}, 3494_{\mathrm{w}}\left(\mathrm{H}_{2} \mathrm{O}\right) ; 1278_{\mathrm{vs}} v_{2}\left(\mathrm{~A}^{\prime}\right), 1014_{\mathrm{m}} v_{3}\left(\mathrm{~A}^{\prime}\right), 836_{\mathrm{m}}$ $v_{6}\left(\mathrm{~A}^{\prime \prime}\right), 769_{\mathrm{m}} v_{4}\left(\mathrm{~A}^{\prime}\right)$ or $v_{5}\left(\mathrm{~A}^{\prime}\right)\left(\mathrm{NO}_{3}{ }^{-}\right)$. The $v_{1}\left(\mathrm{~A}^{\prime}\right)$ symmetric stretching could not be observed because of overlap with other vibrations. Raman $\left(\mathrm{cm}^{-1}\right)$ : $1037_{\mathrm{m}} v_{1}\left(\mathrm{~A}_{1}^{\prime}\right), 1016_{\mathrm{m}} v_{3}\left(\mathrm{~A}^{\prime}\right)$ (solid); $1047_{\mathrm{vs}} v_{1}\left(\mathrm{~A}_{1}^{\prime}\right)$ (aqueous solution). 12b. IR $\left(\mathrm{cm}^{-1}\right.$, solid): $3572_{\mathrm{w}}$ $\left(\mathrm{H}_{2} \mathrm{O}\right) ; 1292_{\mathrm{vs}} v_{2}\left(\mathrm{~A}^{\prime}\right), 1027_{\mathrm{m}} v_{3}\left(\mathrm{~A}^{\prime}\right), 841_{\mathrm{m}} v_{6}\left(\mathrm{~A}^{\prime \prime}\right), 763_{\mathrm{m}} v_{4}\left(\mathrm{~A}^{\prime}\right)$ or $v_{5}\left(\mathrm{~A}^{\prime}\right)\left(\mathrm{NO}_{3}{ }^{-}\right)$. The $v_{1}\left(\mathrm{~A}^{\prime}\right)$ symmetric stretching could not be observed because of overlap with other vibrations.

General Procedure for Coordination Reactions. To a mixture of $\mathbf{3 a}$ or $\mathbf{3 b}(7.7 \mathrm{mg}, 12.5 \mu \mathrm{mol})$ and 11a $(11.5 \mathrm{mg}, 26.0 \mu \mathrm{mol}, 2$ equiv) or 12a (9.5 mg, $26.4 \mu \mathrm{mol}, 2$ equiv) was added acetone- $d_{6}$ $(0.6 \mathrm{~mL})$ and $\mathrm{D}_{2} \mathrm{O}(0.3 \mathrm{~mL})$. The solutions were analyzed by NMR spectroscopy. The products $\mathbf{1 6}-\mathbf{1 9}$ were precipitated by slow diffusion of $\mathrm{Et}_{2} \mathrm{O}$ in the reaction mixture, followed by decantation of the solvents. Drying of the residues in vacuo yielded solid compounds.

cis-[Pt(4- $\{p$-pyridyl $\left.\left.\} \operatorname{dmba}\{[\operatorname{Pt}(\mathrm{NCN})]\}_{2}\right)_{2}\right]\left(\mathrm{NO}_{3}\right)_{2}$ (16). Yield: $15 \mathrm{mg}(10 \mu \mathrm{mol}, 80 \%) .{ }^{1} \mathrm{H}$ NMR $\left(2: 1\right.$ acetone- $\left.d_{6} / \mathrm{D}_{2} \mathrm{O}\right): \delta 2.79(\mathrm{~s}$, $\left.24 \mathrm{H}, \mathrm{NCH}_{3}, \eta^{3}-\mathrm{NCN}\right), 2.88$ (s, 12H, $\left.\mathrm{NCH}_{3}, \eta^{2}-\mathrm{CN}\right), 3.95$ (s, 4H, $\left.\mathrm{CH}_{2} \mathrm{~N}, \eta^{2}-\mathrm{CN}\right), 4.18\left(\mathrm{~s}, 8 \mathrm{H}, \mathrm{CH}_{2} \mathrm{~N}, \eta^{3}-\mathrm{NCN}\right), 6.87\left(\mathrm{~d},{ }^{3} J_{\mathrm{H}, \mathrm{H}}=8\right.$ $\left.\mathrm{Hz}, 4 \mathrm{H}, \mathrm{ArH}-3,5, \eta^{3}-\mathrm{NCN}\right), 6.97\left(\mathrm{t},{ }^{3} J_{\mathrm{H}, \mathrm{H}}=6 \mathrm{~Hz}, 2 \mathrm{H}, \mathrm{ArH}-4, \eta^{3}-\right.$ $\mathrm{NCN}$ ), 7.39-7.50 (m, 4H, ArH-5,6, $\left.\eta^{2}-\mathrm{CN}\right), 7.59$ (s, 2H, ArH-3, $\left.\eta^{2}-\mathrm{CN}\right), 8.01\left(\mathrm{~d},{ }^{3} J_{\mathrm{H}, \mathrm{H}}=5 \mathrm{~Hz}, 4 \mathrm{H}, \operatorname{ArH}-8,11, \eta^{2}-\mathrm{CN}\right), 8.91(\mathrm{~d}$, ${ }^{3} J_{\mathrm{H}, \mathrm{H}}=5 \mathrm{~Hz}, 4 \mathrm{H}$, ArH-9,10, $\left.\eta^{2}-\mathrm{CN}\right) .{ }^{13} \mathrm{C}\left\{{ }^{1} \mathrm{H}\right\} \mathrm{NMR}(0.95: 0.05$ acetone- $\left.d_{6} / \mathrm{D}_{2} \mathrm{O}\right): \delta 50.2\left(\mathrm{NCH}_{3}, \eta^{2}-\mathrm{CN}\right), 53.9\left(\mathrm{NCH}_{3}, \eta^{3}-\mathrm{NCN}\right)$, $73.7\left(\mathrm{CH}_{2} \mathrm{~N}, \eta^{2}-\mathrm{CN}\right), 77.7\left(\mathrm{CH}_{2} \mathrm{~N}, \eta^{3}-\mathrm{NCN}\right), 119.9,123.4,124.2$, $125.4,129.6,139.7,144.4,145.4,147.8,150.9,151.6,151.9 .{ }^{195} \mathrm{Pt}$ NMR (0.95:0.05 acetone- $\left.d_{6} / \mathrm{D}_{2} \mathrm{O}\right): \delta-3331.2,-3381.9$. Anal. Calcd for $\mathrm{C}_{52} \mathrm{H}_{68} \mathrm{~N}_{10} \mathrm{O}_{6} \mathrm{Pt}_{3}$ : C, 41.24; H, 4.53; N, 9.25. Found: $\mathrm{C}$, 41.15; H, 4.47; N, 9.19.

cis-[Pt(4- $\{p$-pyridyl $\left.\} \operatorname{dmba}\left\{[\operatorname{Pd}(\mathrm{NCN})\}_{2}\right)_{2}\right]\left(\mathrm{NO}_{3}\right)_{2}$ (17). Yield: $12 \mathrm{mg}(9 \mu \mathrm{mol}, 72 \%) .{ }^{1} \mathrm{H}$ NMR $\left(2: 1\right.$ acetone- $\left.d_{6} / \mathrm{D}_{2} \mathrm{O}\right): \delta 2.64(\mathrm{~s}$,

(12) Terheijden, J.; van Koten, G.; Muller, F.; Grove, D. M.; Vrieze, K.; Nielsen, E.; Stam, C. H. J. Organomet. Chem. 1986, 315, 401-417. 
Amijs et al.

Table 1. Crystallographic Details

\begin{tabular}{|c|c|c|c|c|}
\hline compd & 3a & 11a & $11 \mathrm{a} \cdot \mathrm{H}_{2} \mathrm{O}$ & 16 \\
\hline formula & $\mathrm{C}_{28} \mathrm{H}_{30} \mathrm{~N}_{4} \mathrm{Pt}$ & $\mathrm{C}_{12} \mathrm{H}_{19} \mathrm{~N}_{3} \mathrm{O}_{3} \mathrm{Pt}$ & $\mathrm{C}_{12} \mathrm{H}_{19} \mathrm{~N}_{3} \mathrm{O}_{3} \mathrm{Pt} \cdot \mathrm{H}_{2} \mathrm{O}$ & {$\left[\mathrm{C}_{52} \mathrm{H}_{68} \mathrm{~N}_{8} \mathrm{Pt}_{3}\right]\left(\mathrm{NO}_{3}\right)_{2}+$ disordered solvent } \\
\hline fw & 617.65 & 448.39 & 466.41 & $1514.43^{a}$ \\
\hline cryst color & yellowish & colorless & colorless & yellowish \\
\hline cryst size $\left[\mathrm{mm}^{3}\right]$ & $0.42 \times 0.18 \times 0.06$ & $0.24 \times 0.09 \times 0.06$ & $0.36 \times 0.30 \times 0.09$ & $0.42 \times 0.12 \times 0.04$ \\
\hline cryst syst & triclinic & orthorhombic & triclinic & monoclinic \\
\hline space group & $P \overline{1}($ No. 2$)$ & $\operatorname{Pna2}_{1}$ (No. 33) & $P \overline{1}$ (No. 2$)$ & $P 2{ }_{1} / c$ (No. 14) \\
\hline$a[\AA]$ & $9.1884(10)$ & $10.5554(4)$ & $8.8915(3)$ & $18.1883(3)$ \\
\hline$b[\AA]$ & $10.7555(17)$ & $12.5362(4)$ & $9.0104(2)$ & $21.1087(4)$ \\
\hline$c[\AA]$ & $12.6573(10)$ & $10.8290(2)$ & $9.6808(2)$ & $23.0192(4)$ \\
\hline$\alpha[\mathrm{deg}]$ & $98.903(7)$ & 90 & $77.947(2)$ & 90 \\
\hline$\beta$ [deg] & $103.582(6)$ & 90 & $87.790(2)$ & 128.2015(6) \\
\hline$\gamma[\mathrm{deg}]$ & $92.374(10)$ & 90 & $77.378(2)$ & 90 \\
\hline$V\left[\AA^{3}\right]$ & 1197.3(3) & 1432.94(8) & 740.14(3) & 6945.1(2) \\
\hline$Z$ & 2 & 4 & 2 & 4 \\
\hline$D_{x}\left[\mathrm{~g} / \mathrm{cm}^{3}\right]$ & 1.713 & 2.078 & 2.093 & $1.448^{a}$ \\
\hline$\mu\left[\mathrm{mm}^{-1}\right]$ & 5.883 & 9.800 & 9.495 & $6.072^{a}$ \\
\hline abs corr & multiscan & analytical & analytical & analytical \\
\hline abs corr range & $0.29-0.70$ & $0.19-0.55$ & $0.08-0.48$ & $0.21-0.81$ \\
\hline refln measd/unique & $17000 / 5481$ & $11035 / 3247$ & $18901 / 3401$ & $70031 / 10269$ \\
\hline$(\sin \theta / \lambda)_{\max }\left[\AA^{-1}\right]$ & 0.65 & 0.65 & 0.65 & 0.56 \\
\hline param/restraints & $302 / 58$ & $176 / 1$ & $193 / 0$ & $612 / 32$ \\
\hline $\mathrm{R} 1 / \mathrm{wR} 2[I>2 \sigma(I)]$ & $0.0267 / 0.0641$ & $0.0214 / 0.0443$ & $0.0134 / 0.0331$ & $0.0405 / 0.1074$ \\
\hline $\mathrm{R} 1 / \mathrm{wR} 2$ [all reflns] & $0.0326 / 0.0664$ & $0.0273 / 0.0464$ & $0.0142 / 0.0334$ & $0.0567 / 0.1138$ \\
\hline$S$ & 1.085 & 1.032 & 1.190 & 1.080 \\
\hline Flack $x$ parameter ${ }^{16}$ & & $-0.013(9)$ & & \\
\hline$\rho_{\min / \max }\left[\mathrm{e} / \AA^{3}\right]$ & $-1.24 / 1.51$ & $-1.31 / 0.70$ & $-1.27 / 0.42$ & $-0.73 / 2.46$ \\
\hline
\end{tabular}

${ }^{a}$ Derived quantities do not contain the contribution of the disordered solvent molecules.

$\left.24 \mathrm{H}, \mathrm{NCH}_{3}, \eta^{3}-\mathrm{NCN}\right), 2.78\left(\mathrm{~s}, 12 \mathrm{H}, \mathrm{NCH}_{3}, \eta^{2}-\mathrm{CN}\right), 3.93$ (s, 4H, $\left.\mathrm{CH}_{2} \mathrm{~N}, \eta^{2}-\mathrm{CN}\right), 4.04\left(\mathrm{~s}, 8 \mathrm{H}, \mathrm{CH}_{2} \mathrm{~N}, \eta^{3}-\mathrm{NCN}\right), 6.80\left(\mathrm{~d},{ }^{3} J_{\mathrm{H}, \mathrm{H}}=7\right.$ $\left.\mathrm{Hz}, 4 \mathrm{H}, \mathrm{ArH}-3,5, \eta^{3}-\mathrm{NCN}\right), 6.96\left(\mathrm{t},{ }^{3} J_{\mathrm{H}, \mathrm{H}}=7 \mathrm{~Hz}, 2 \mathrm{H}, \mathrm{ArH}-4, \eta^{3}-\right.$ $\mathrm{NCN}$ ), 7.36-7.53 (m, 6H, ArH, $\left.\eta^{2}-\mathrm{CN}\right), 7.87$ (bs, 4H, ArH-8,11, $\left.\eta^{2}-\mathrm{CN}\right), 8.81$ (bs, 4H, ArH-9,10, $\left.\eta^{2}-\mathrm{CN}\right) .{ }^{13} \mathrm{C}\left\{{ }^{1} \mathrm{H}\right\}$ NMR $(2: 1$ acetone- $\left.d_{6} / \mathrm{D}_{2} \mathrm{O}\right): \delta 50.1,\left(\mathrm{NCH}_{3}, \eta^{2}-\mathrm{CN}\right), 52.2\left(\mathrm{NCH}_{3}, \eta^{3}-\mathrm{NCN}\right)$, $73.5\left(\mathrm{CH}_{2} \mathrm{~N}, \eta^{2}-\mathrm{CN}\right), 74.3\left(\mathrm{CH}_{2} \mathrm{~N}, \eta^{3}-\mathrm{NCN}\right), 116.8,120.2,120.6$, 122.5, 124.2, 125.9, 130.7, 136.2, 139.6, 146.3, 150.9, 151.1. Anal. Calcd for $\mathrm{C}_{52} \mathrm{H}_{68} \mathrm{~N}_{10} \mathrm{O}_{6} \mathrm{Pd}_{2} \mathrm{Pt}$ : C, 46.71; H, 5.13; N, 10.48. Found: C, 46.63; H, 4.97; N, 10.33 .

cis-[Pt $\left(4-\{m\right.$-pyridyl $\left.\} \operatorname{dmba}\left\{[\mathbf{P t}(\mathbf{N C N})]_{2}\right)_{2}\right]\left(\mathbf{N O}_{3}\right)_{2}$ (18). Yield: $14 \mathrm{mg}(9 \mu \mathrm{mol}, 72 \%) .{ }^{1} \mathrm{H}$ NMR $\left(2: 1\right.$ acetone- $\left.d_{6} / \mathrm{D}_{2} \mathrm{O}\right): \delta 2.79(\mathrm{~s}$, $\left.12 \mathrm{H}, \mathrm{NCH}_{3}, \eta^{2}-\mathrm{CN}\right), 2.82$ (s, 24H, $\left.\mathrm{NCH}_{3}, \eta^{3}-\mathrm{NCN}\right), 3.93$ (s, $4 \mathrm{H}$, $\left.\mathrm{CH}_{2} \mathrm{~N}, \eta^{2}-\mathrm{CN}\right), 4.20\left(\mathrm{~s}, 8 \mathrm{H}, \mathrm{CH}_{2} \mathrm{~N}, \eta^{3}-\mathrm{NCN}\right), 6.88\left(\mathrm{~d},{ }^{3} J_{\mathrm{H}, \mathrm{H}}=8\right.$ $\left.\mathrm{Hz}, 4 \mathrm{H}, \mathrm{ArH}-3,5, \eta^{3}-\mathrm{NCN}\right), 6.99\left(\mathrm{t},{ }^{3} J_{\mathrm{H}, \mathrm{H}}=7 \mathrm{~Hz}, 2 \mathrm{H}, \mathrm{ArH}-4, \eta^{3}-\right.$ $\mathrm{NCN}), 7.28\left(\mathrm{~d},{ }^{3} J_{\mathrm{H}, \mathrm{H}}=8 \mathrm{~Hz}, 2 \mathrm{H}, \mathrm{ArH}, \eta^{2}-\mathrm{CN}\right), 7.46(\mathrm{~m}, 4 \mathrm{H}, \mathrm{ArH}$, $\left.\eta^{2}-\mathrm{CN}\right), 7.80\left(\mathrm{t},{ }^{3} J_{\mathrm{H}, \mathrm{H}}=5 \mathrm{~Hz}, 2 \mathrm{H}, \mathrm{ArH}-10, \eta^{2}-\mathrm{CN}\right), 8.29\left(\mathrm{~d},{ }^{3} J_{\mathrm{H}, \mathrm{H}}\right.$ $\left.=8 \mathrm{~Hz}, 2 \mathrm{H}, \mathrm{ArH}-11, \eta^{2}-\mathrm{CN}\right), 8.91\left(\mathrm{~d},{ }^{3} J_{\mathrm{H}, \mathrm{H}}=5 \mathrm{~Hz}, 2 \mathrm{H}\right.$, ArH-9, $\eta^{2}-\mathrm{CN}$ ), 9.21 (s, 2H, ArH-8). ${ }^{13} \mathrm{C}\left\{{ }^{1} \mathrm{H}\right\}$ NMR (0.95:0.05 acetone$\left.d_{6} / \mathrm{D}_{2} \mathrm{O}\right): \delta 50.3\left(\mathrm{NCH}_{3}, \eta^{2}-\mathrm{CN}\right), 54.0\left({ }^{3} J_{\mathrm{Pt}, \mathrm{C}}=61.1 \mathrm{~Hz}, \mathrm{NCH}_{3}\right.$, $\left.\eta^{3}-\mathrm{NCN}\right), 73.9\left(\mathrm{CH}_{2} \mathrm{~N}, \eta^{2}-\mathrm{CN}\right), 77.8\left(\mathrm{CH}_{2} \mathrm{~N}, \eta^{3}-\mathrm{NCN}\right), 120.1$, 120.3, 124.5, 125.6, 127.9, 129.8, 136.7, 139.7, 139.9, 141.4, 144.2, 145.0, 145.6, 149.1, 149.6, 150.7. Anal. Calcd for $\mathrm{C}_{52} \mathrm{H}_{68} \mathrm{~N}_{10} \mathrm{O}_{6}$ $\mathrm{Pt}_{3}$ : C, 41.24; H, 4.53; N, 9.25. Found: C, 41.06; H, 4.61; N, 9.18.

cis-[Pt(4- $\{m$-pyridyl $\}$ dmba $\left.\left.\{[\mathbf{P d}(\mathbf{N C N})]\}_{2}\right)_{2}\right]\left(\mathbf{N O}_{3}\right)_{2}$ (19). Yield: $11 \mathrm{mg}(8 \mu \mathrm{mol}, 64 \%) .{ }^{1} \mathrm{H}$ NMR $\left(2: 1\right.$ acetone- $\left.d_{6} / \mathrm{D}_{2} \mathrm{O}\right): \delta 2.71(\mathrm{~s}$, $\left.24 \mathrm{H}, \mathrm{NCH}_{3}, \eta^{3}-\mathrm{NCN}\right), 2.77$ (s, 12H, $\left.\mathrm{NCH}_{3}, \eta^{2}-\mathrm{CN}\right), 3.90$ (s, 4H, $\left.\mathrm{CH}_{2} \mathrm{~N}, \eta^{2}-\mathrm{CN}\right), 4.05\left(\mathrm{~s}, 8 \mathrm{H}, \mathrm{CH}_{2} \mathrm{~N}, \eta^{3}-\mathrm{NCN}\right), 6.80\left(\mathrm{~d},{ }^{3} J_{\mathrm{H}, \mathrm{H}}=7\right.$ $\left.\mathrm{Hz}, 4 \mathrm{H}, \mathrm{ArH}-3,5, \eta^{3}-\mathrm{NCN}\right), 6.96\left(\mathrm{t},{ }^{3} J_{\mathrm{H}, \mathrm{H}}=8 \mathrm{~Hz}, 2 \mathrm{H}, \mathrm{ArH}-4, \eta^{3}-\right.$ $\mathrm{NCN}$ ), 7.24-7.42 (m, 6H, ArH, $\left.\eta^{2}-\mathrm{CN}\right), 7.62$ (bs, 2H, ArH-10, $\eta^{2}$-CN), 8.17 (bs, 2H, ArH-11, $\left.\eta^{2}-\mathrm{CN}\right), 8.74$ (bs, 2H, ArH-9, $\eta^{2}$ $\mathrm{CN}), 9.09$ (bs, 2H, ArH-8, $\left.\eta^{2}-\mathrm{CN}\right) .{ }^{13} \mathrm{C}\left\{{ }^{1} \mathrm{H}\right\}$ NMR (0.95:0.05 acetone- $\left.d_{6} / \mathrm{D}_{2} \mathrm{O}\right): \delta 50.3\left(\mathrm{NCH}_{3}, \eta^{2}-\mathrm{CN}\right), 52.3\left({ }^{3} J_{\mathrm{Pt}, \mathrm{C}}=61 \mathrm{~Hz}\right.$, $\left.\mathrm{NCH}_{3}, \eta^{3}-\mathrm{NCN}\right), 73.9\left(\mathrm{CH}_{2} \mathrm{~N}, \eta^{2}-\mathrm{CN}\right), 74.5\left(\mathrm{CH}_{2} \mathrm{~N}, \eta^{3}-\mathrm{NCN}\right)$, 120.2, 120.2, 124.6, 125.8, 128.1, 129.6, 136.9, 139.7, 139.9, 141.5, 144.1, 144.9, 145.6, 149.2, 149.8, 150.9. Anal. Calcd for $\mathrm{C}_{52} \mathrm{H}_{68} \mathrm{~N}_{10} \mathrm{O}_{6}$ $\mathrm{Pd}_{2} \mathrm{Pt}$ : C, 46.71; H, 5.13; N, 10.48. Found: C, 46.54; H, 5.06; N, 10.36 .
Crystal Structure Determinations. X-ray intensities were measured on a Nonius Kappa CCD diffractometer with rotatinganode and graphite monochromators $(\lambda=0.71073 \AA)$ at a temperature of $150(2) \mathrm{K}$. The structures were solved with automated Patterson methods ${ }^{13}$ and refined with SHELXL-97 ${ }^{14}$ on $F^{2}$ of all reflections. Non-hydrogen atoms were refined freely with anisotropic displacement parameters. Hydrogen atoms were introduced in calculated positions and refined as rigid groups. The crystal structure of 16 contains large voids (1957.1 $\AA^{3} /$ unit cell) filled with disordered solvent molecules. Their contribution to the structure factors was secured by Fourier backtransformation using the routine SQUEEZE of the PLATON ${ }^{15}$ package resulting in 636 electrons/ unit cell.

Geometry calculations, drawings, and checks for higher symmetry were performed with the PLATON ${ }^{15}$ package. Further crystallographic details are given in Table 1.

\section{Results and Discussion}

Synthesis and Characterization of Pyridyl-Functionalized Organoplatinum Complexes. Platination of orthochelating aminoaryl ligands is generally achieved via a sequence of two reactions involving the regioselective ortholithiation of the ligand first, followed by subsequent transmetalation with a cis- or trans- $\left[\mathrm{PtX}_{2} \mathrm{~L}_{2}\right]$ salt $(\mathrm{X}=\mathrm{Br}, \mathrm{Cl}, \mathrm{L}$ $\left.=\operatorname{cod}, \mathrm{SEt}_{2}, \mathrm{SMe}_{2}\right){ }^{5,17}$ Initially, this route was applied for the synthesis of pyridyl-functionalized $\mathrm{C}, \mathrm{N}$-ortho-chelated

(13) Beurskens, P. T.; Admiraal, G.; Beurskens, G.; Bosman, W. P.; GarciaGranda, S.; Gould, R. O.; Smits, J. M. M.; Smykalla, C. The DIRDIF99 program system; Technical Report of the Crystallography Laboratory at University of Nijmegen; University of Nijmegen: Nijmegen, The Netherlands, 1999.

(14) Sheldrick, G. M. SHELXL-97. Program for crystal structure refinement; Universität Göttingen: Göttingen, Germany, 1997.

(15) Spek, A. L. J. Appl. Crystallogr. 2003, 36, 7-13.

(16) Flack, H. D. Acta Crystallogr. 1983, A39, 876-881.

(17) Wehman-Ooyevaar, I. C. M.; Grove, D. M.; Kooijman, H.; van de Sluis, P.; Spek, A. L.; van Koten, G. J. Am. Chem. Soc. 1992, 114, 9916-9924. 
Scheme 1. Attempted Synthesis of 4-Pyridyl-Functionalized Corner Building Block 3a ${ }^{a}$
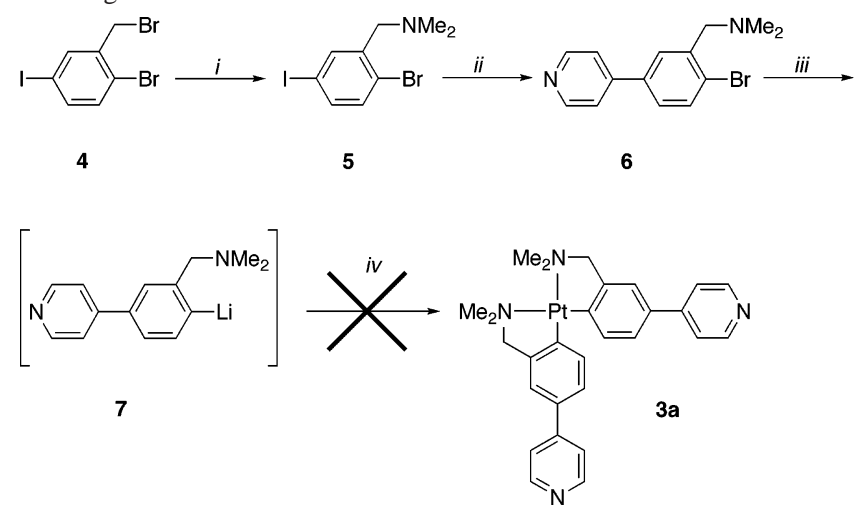

a (i) $\mathrm{HNMe}_{2}, \mathrm{Et}_{2} \mathrm{O}, \mathrm{RT}, 2$ h. (ii) 4-Pyridylborane pinacol ester, [PdCl $\mathrm{Pdppf}_{2}$ ), $\mathrm{Na}_{2} \mathrm{CO}_{3}, \mathrm{DME} / \mathrm{THF} / \mathrm{H}_{2} \mathrm{O}, \Delta \mathrm{T}, 40$ h. (iii) $n-\mathrm{BuLi}, \mathrm{Et}_{2} \mathrm{O},-78$ ${ }^{\circ} \mathrm{C}, 10$ min. (iv) $\left[\mathrm{PtCl}_{2}(\operatorname{cod})\right], \mathrm{Et}_{2} \mathrm{O},-78{ }^{\circ} \mathrm{C} \rightarrow \mathrm{RT}, 3 \mathrm{~h}$.

aminoaryl platinum(II) corner complexes, starting from 1-bromo-2-(bromomethyl)-4-iodobenzene (4) ${ }^{7}$ (Scheme 1). The positions of the iodide and bromide substituents were chosen such that the para-position could be selectively addressed by a Suzuki-Miyaura $\mathrm{C}-\mathrm{C}$ coupling reaction with 4-pyridylborane pinacol ester because of the higher reactivity of the $\mathrm{C}-\mathrm{I}$ bond over the corresponding $\mathrm{C}-\mathrm{Br}$ bond in $\mathrm{C}-\mathrm{C}$ cross-coupling reactions. The Suzuki-Miyaura coupling reaction yielded $\mathbf{6}$ as an oil after purification via column chromatography in $65 \%$ yield. Chemoselective lithiation of 6 at the $\mathrm{C}-\mathrm{Br}$ position was achieved by reacting 6 with 1 equiv of $n$ - BuLi in $\mathrm{Et}_{2} \mathrm{O}$ at $-78{ }^{\circ} \mathrm{C}$ for $10 \mathrm{~min}$. Quenching an aliquot of the reaction mixture with $\mathrm{S}_{2} \mathrm{Me}_{2}$, followed by analysis with ${ }^{1} \mathrm{H}$ NMR and GC-MS spectroscopy, confirmed that complete exchange of the bromo substituent for a methyl sulfide group had occurred. Under these reaction conditions, no trace of products resulting from the reaction of $n$-BuLi with the pyridyl ring was observed. However, the platination reaction of intermediate lithium complex 7 with 0.5 equiv of $\left[\mathrm{PtCl}_{2}(\mathrm{cod})\right]$ did not result in the formation of the cisplatinum(II) complex 3a. Instead, the protonated ligand was obtained after workup. Variation in the reaction temperatures $\left(-10{ }^{\circ} \mathrm{C}\right.$ to reflux) and prolonged reaction times did not improve this result.

The alternative route toward the synthesis of functionalized organometallic complexes would involve the synthesis of the organometallic complex containing metal-carbon bonds prior to the subsequent organic functionalization reaction, via either lithiation or a metal-catalyzed $\mathrm{C}-\mathrm{C}$ bond formation reaction. These kinds of nucleophilic substitution reactions are rarely performed on organometallic complexes, and the lithiation reactions have thus far been reported by Roper and co-workers and our group only.,18,19 Furthermore, a few examples of $\mathrm{C}-\mathrm{C}$ cross-coupling reactions on organometallic

(18) (a) Clark, A. M.; Richard, C. E. F.; Roper, W. R.; Wright, L. J. Organometallics 1998, 17, 4535-4537. (b) Clark, A. M.; Richard, C. E. F.; Roper, W. R.; Wright, L. J. Organometallics 1999, 18, 28132820. (c) Slagt, M. Q.; Klein Gebbink, R. J. M.; Lutz, M.; Spek, A. L.; van Koten, G. J. Chem. Soc., Dalton Trans. 2002, 2591-2592.

(19) Rodríguez, G.; Albrecht, M.; Schoenmaker, J.; Ford, A.; Lutz, M.; Spek, A. L.; van Koten, G. J. Am. Chem. Soc. 2002, 124, 51275138.
Scheme 2. Synthesis of Pyridyl-Functionalized Corner Molecules 3a and $\mathbf{3} \mathbf{b}^{a}$
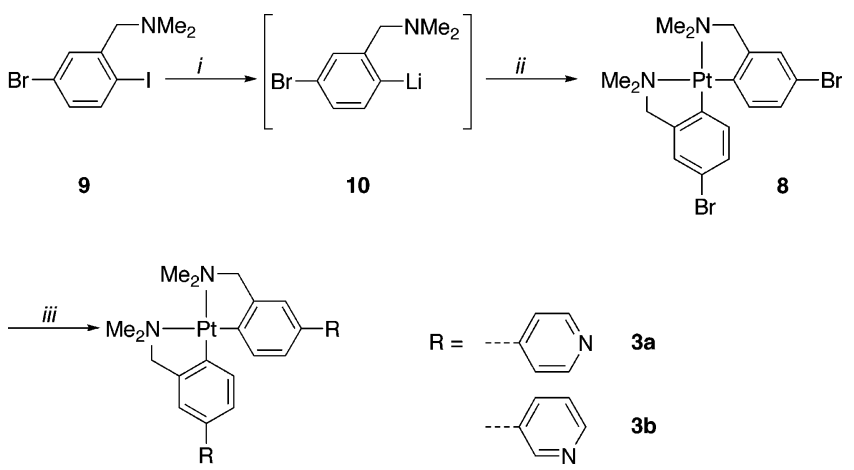

a (i) $n$ - $\mathrm{BuLi}, \mathrm{Et}_{2} \mathrm{O},-78{ }^{\circ} \mathrm{C}, 10$ min. (ii) cis- $\left[\mathrm{PtCl}_{2}(\operatorname{cod})\right], \mathrm{Et}_{2} \mathrm{O},-78{ }^{\circ} \mathrm{C}$, $\mathrm{RT}, 3$ h. (iii) 4- or 3-pyridylborane pinacol ester (a and $\mathbf{b}$, respectively), $\left[\mathrm{PdCl}_{2}(\mathrm{dppf})\right], \mathrm{Na}_{2} \mathrm{CO}_{3}, \mathrm{DME} / \mathrm{THF} / \mathrm{H}_{2} \mathrm{O}, 70{ }^{\circ} \mathrm{C}, 3 \mathrm{~h}$.

complexes have been reported, i.e., Suzuki-Miyaura and Sonogashira reactions on organoosmium(IV) and -ruthenium(II) complexes. $^{20} \mathrm{We}$ recently reported the palladiumcatalyzed Suzuki-Miyaura cross-coupling reaction of a SCSpincer borane compound $\left(\mathrm{SCS}=\left[\mathrm{C}_{6} \mathrm{H}_{3}\left(\mathrm{CH}_{2} \mathrm{~S} t \mathrm{Bu}\right)_{2}-1,3\right]^{-}\right)$ with bromide-functionalized $\left[\mathrm{Pt}(\mathrm{C}, \mathrm{N})_{2}\right]$ complex $8{ }^{6}$ Following the same reaction procedure (Scheme 2), the synthesis of pyridyl-substituted $\left[\mathrm{Pt}(\mathrm{C}, \mathrm{N})_{2}\right](\mathrm{C}, \mathrm{N}=C, N$-ortho-chelating ligand) complexes $\mathbf{3}$ could be achieved. Complex $\mathbf{8}$, synthesized in $92 \%$ yield by the reaction of 0.5 equiv of $\left[\mathrm{PtCl}_{2}-\right.$ (cod)] with lithium complex 10, is stable under basic conditions, which allows it to be used in a subsequent Suzuki-Miyaura coupling reaction. The reaction of $\mathbf{8}$ with either 4- or 3-pyridylborane pinacol ester in a mixture of DME, THF, and $\mathrm{H}_{2} \mathrm{O}(1: 1: 1)$, with $\mathrm{Na}_{2} \mathrm{CO}_{3}$ and $\left[\mathrm{PdCl}_{2}\right.$ (dppf)] (dppf $=($ diphenylphosphino)ferrocene $)$ as the catalysts, was completed at $70{ }^{\circ} \mathrm{C}$ within $3 \mathrm{~h}$. It is noteworthy that no decomposition of $\mathbf{8}$ was observed and conversion to $c i s-[\mathrm{Pt}-$ (4-\{p-pyridyl $\}$-dmba $\left.)_{2}\right]$ (3a) and cis-[Pt(4-\{m-pyridyl $\}$ $\mathrm{dmba})_{2}$ ] (3b) was quantitative (Scheme 2). Purification of the reaction mixtures by column chromatography afforded 3a and $\mathbf{3 b}$ in moderate to good yields as air- and moisturestable, light-yellow-colored solids. No cis-to-trans isomerization of the complexes was observed during the reactions or purification of the products.

Proof of the cis configuration of $\mathbf{3}$ was provided by the X-ray crystal structure of $\mathbf{3 a}$. Single crystals of $\mathbf{3 a}$ suitable for an X-ray crystal structure determination were obtained by slow vapor diffusion of $\mathrm{Et}_{2} \mathrm{O}$ into a saturated solution of 3a in $\mathrm{CH}_{2} \mathrm{Cl}_{2}$. The molecular structure is depicted in Figure 1 , and relevant bond lengths, bond angles, and torsion angles are listed in Table 2. The molecular structure of 3a shows a slightly distorted square-planar platinum center bonded to two bidentate, $C, N$-chelating aminoaryl ligands in a cis configuration. This is reflected by the acute bite angles of the two $C, N$ ligands at the platinum center, $80.44(14)^{\circ}(\mathrm{N} 11-$ $\mathrm{Pt} 1-\mathrm{C} 11)$ and $81.16(14)^{\circ}(\mathrm{N} 21-\mathrm{Pt} 1-\mathrm{C} 21)$. These values are comparable to $\mathrm{N}-\mathrm{Pt}-\mathrm{C}$ angles of similar $\left[\right.$ cis- $\left.\mathrm{Pt}(\mathrm{C}, \mathrm{N})_{2}\right]$

(20) (a) Chodorowski-Kimmes, S.; Beley, M.; Collin, J.-P.; Sauvage, J.-P. Tetrahedron Lett. 1996, 37, 2963-2966. (b) Fraysse, S.; Coudret, C.; Launay, J.-P. J. Am. Chem. Soc. 2003, 125, 5880-5888. (c) Miyaura, N.; Yanagi, T.; Suzuki, A. Synth. Commun. 1981, 11, 513. 


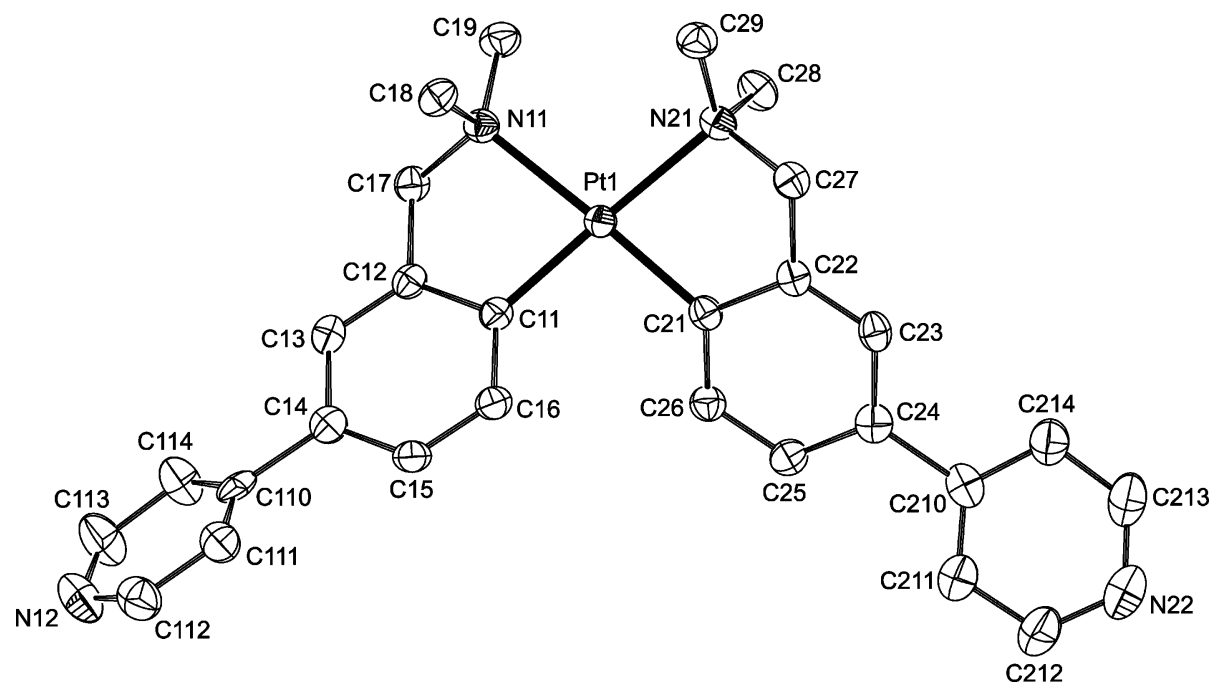

Figure 1. Displacement ellipsoid plot (50\% probability level) of the molecular structure of 3a in the crystal, with the adopted labeling scheme. Hydrogen atoms have been omitted for clarity.

Table 2. Selected Bond Lengths ( $\AA$ ), Bond Angles (deg), and Torsion Angles (deg) for $\mathbf{3 a}$

\begin{tabular}{|c|c|c|c|}
\hline \multicolumn{4}{|c|}{ Interatomic Distances } \\
\hline Pt1-N11 & 2.191(3) & $\mathrm{Pt} 1-\mathrm{N} 21$ & $2.202(3)$ \\
\hline $\mathrm{Pt} 1-\mathrm{C} 11$ & $1.980(4)$ & $\mathrm{Pt} 1-\mathrm{C} 21$ & $1.981(4)$ \\
\hline \multicolumn{4}{|c|}{ Interatomic Angles and Torsion Angles } \\
\hline $\mathrm{N} 11-\mathrm{Pt} 1-\mathrm{N} 21$ & $102.39(12)$ & $\mathrm{C} 11-\mathrm{Pt} 1-\mathrm{C} 21$ & $97.28(15)$ \\
\hline $\mathrm{N} 11-\mathrm{Pt} 1-\mathrm{C} 11$ & $80.44(14)$ & $\mathrm{N} 21-\mathrm{Pt} 1-\mathrm{C} 21$ & $81.16(14)$ \\
\hline $\mathrm{N} 11-\mathrm{Pt} 1-\mathrm{C} 21$ & 171.04(14) & $\mathrm{N} 21-\mathrm{Pt} 1-\mathrm{C} 11$ & $171.21(14)$ \\
\hline $\mathrm{N} 11-\mathrm{Pt} 1-\mathrm{C} 11-\mathrm{C} 12$ & $15.4(3)$ & $\mathrm{N} 21-\mathrm{Pt} 1-\mathrm{C} 21-\mathrm{C} 22$ & $12.2(3)$ \\
\hline $11-\mathrm{Pt} 1-\mathrm{C} 21-\mathrm{C} 26$ & $29.8(4)$ & $\mathrm{C} 21-\mathrm{Pt} 1-\mathrm{C} 11-\mathrm{C} 16$ & $32.8(4)$ \\
\hline & 33 & 2 & 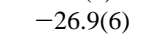 \\
\hline
\end{tabular}

complexes $\left(79.57-80.97^{\circ}\right) .{ }^{6}$ The two five-membered metallacycles are puckered, which is shown by the torsion angles of $15.4(3)^{\circ}$ and $12.2(3)^{\circ}$ for $\mathrm{N} 11-\mathrm{Pt} 1-\mathrm{C} 11-\mathrm{C} 12$ and $\mathrm{N} 21-$ $\mathrm{Pt} 1-\mathrm{C} 21-\mathrm{C} 22$, respectively. The torsion angles around the platinum center are $32.9(4)^{\circ}$ and $29.8(4)^{\circ}$ for $\mathrm{C} 21-\mathrm{Pt} 1-$ $\mathrm{C} 11-\mathrm{C} 16$ and $\mathrm{C} 11-\mathrm{Pt} 1-\mathrm{C} 21-\mathrm{C} 26$, respectively, showing that the structure is not completely flat (see Figure 4 ). The angles between the aryl and pyridyl rings of $33.5(6)^{\circ}$ and $-26.9(6)^{\circ}(\mathrm{C} 15-\mathrm{C} 14-\mathrm{C} 110-\mathrm{C} 111$ and $\mathrm{C} 25-\mathrm{C} 24-\mathrm{C} 210-$ $\mathrm{C} 211$, respectively) fall in the range normally observed for similar compounds. ${ }^{6}$ In the crystal of $\mathbf{3 a}$, there are short intermolecular contacts between the platinum center and the benzylic hydrogen atoms H17A and H27B, respectively, with distances of 2.81 and $2.92 \AA$, which is shorter than the sum of their contact radii.

Only one set of signals for both aminoaryl ligands was observed in the ${ }^{1} \mathrm{H}$ NMR spectra of $\mathbf{3}$ in $\mathrm{CD}_{2} \mathrm{Cl}_{2}$, indicating that in solution both complexes have an apparent $C_{2 v}$ symmetry on the NMR time scale. In the ${ }^{1} \mathrm{H}$ NMR spectra of $\mathbf{3 a}$ and $\mathbf{3} \mathbf{b}$, singlets for $\mathrm{CH}_{2} \mathrm{NMe}_{2}$ protons with low ${ }^{3} J_{\mathrm{Pt}-\mathrm{H}}$ couplings constants $(11-16 \mathrm{~Hz})$ were observed (Table 6), which confirmed the anticipated cis configuration of $\mathbf{3} \mathbf{a}$ and 3b. ${ }^{5,6}$ Variable-temperature ${ }^{1} \mathrm{H}$ NMR experiments in the range of room temperature to $-80{ }^{\circ} \mathrm{C}$ did not result in decoalescence of the singlet resonances of the benzylic protons, which demonstrates that even at low temperatures the interconversion process between the two possible diastereomers $\Delta$ and $\Lambda$ is fast on the NMR time scale, as was already observed for precursor $\mathbf{8}{ }^{6}$ The flexibility of $\mathbf{3 a}$ and $\mathbf{3 b}$ points to the possibility of using these complexes as flat corner molecules in closed molecular structures.

Synthesis and Characterization of $\left[\mathrm{M}(\mathrm{NCN})\left(\mathrm{NO}_{3}\right)\right]$ Complexes. To test the coordinative properties of the pyridyl nitrogen atoms in $\mathbf{3 a}, \mathbf{b}$, complexation reactions with platinum and palladium NCN complexes were performed. Terdentate coordinating, monoanionic ECE-pincer ligand complexes (E $=\mathrm{SR}, \mathrm{NR}_{2}$, and $\mathrm{PR}_{2}$ ) have been used in self-assembly reactions with Lewis bases to form coordination complexes, ${ }^{21}$ polymers, ${ }^{22}$ a triangle, ${ }^{23}$ and organometallic dendrimers. ${ }^{24}$ Recently, the use of the strong coordination bond between a NCN-pincer platinum(II) cation and pyridine in a templateassisted synthesis of heteromacrocycles has been reported. ${ }^{10}$ Because the strength of complexation of a pyridine ligand to a metal center depends on the metal being used, ${ }^{21 \mathrm{c}}$ we have chosen to use both cationic platinum(II) and palladium(II) pincer complexes, $\left[\mathrm{M}(\mathrm{NCN})\left(\mathrm{NO}_{3}\right)\right](\mathrm{M}=\mathrm{Pt}(\mathbf{1 1 a}), \mathrm{Pd}$ (12a)). These complexes were prepared from $[\mathrm{MCl}(\mathrm{NCN})]$ $(\mathrm{M}=\mathrm{Pt}(\mathbf{1 3}), \mathrm{Pd}(\mathbf{1 4})$, respectively) via halide abstraction with silver nitrate (Scheme 3 ), according to the procedure reported for the synthesis of $\left[\mathrm{M}(\mathrm{NCN})\left(\mathrm{OH}_{2}\right)\right]\left(\mathrm{BF}_{4}\right)(\mathrm{M}=$ Pt (11c), Pd (12c); Chart 2). ${ }^{25}$

(21) (a) van Manen, H.-J.; Nakashima, K.; Shinkai, S.; Kooijman, H.; Spek, A. L.; van Veggel, F. C. J. M.; Reinhoudt, D. N. Eur. J. Inorg. Chem. 2000, 2533-2540. (b) Jude, H.; Krause Bauer, J. A.; Connick, W. B. Inorg. Chem. 2004, 43, 725-733. (c) Dijkstra, H. P.; Chuchuryukin, A.; Suijkerbuijk, B. M. J. M.; van Klink, G. P. M.; Mills, A. M.; Spek, A. L.; van Koten, G. Adv. Synth. Catal. 2002, 344, 771-780. (d) Jude, H.; Krause Bauer, J. A.; Connick, W. B. Inorg. Chem. 2005 , 44, 1211-1220

(22) (a) Yount, W. C.; Juwarker, H.; Craig, S. L. Polym. Rep. 2003, 44, 521-522. (b) Yount, W. C.; Juwarker, H.; Craig, S. L. J. Am. Chem. Soc. 2003, 125, 15302-15303.

(23) Hall, J. R.; Loeb, S. J.; Shimizu, G. K. H.; Yap, G. P. A. Angew. Chem., Int. Ed. 1998, 37, 121-123.

(24) (a) Huck, W. T. S.; van Veggel, F. C. J. M.; Kropman, B. L.; Blank, D. H. A.; Keim, E. G.; Smithers, M. M. A.; Reinhoudt, D. N. J. Am. Chem. Soc. 1995, 117, 8293-8294. (b) Huck, W. T. S.; Hulst, R. Timmerman, P.; van Veggel, F. C. J. M.; Reinhoudt, D. N. Angew. Chem., Int. Ed. Engl. 1997, 36, 1006-1008.

(25) Grove, D. M.; van Koten, G.; Louwen, J.; Noltes, J. G.; Spek, A. L.; Ubbels, H. J. C. J. Am. Chem. Soc. 1982, 104, 6609-6616. 
Scheme 3. Synthesis of Cationic Pincer Nitrate Complexes 11a and $12 \mathbf{a}^{a}$

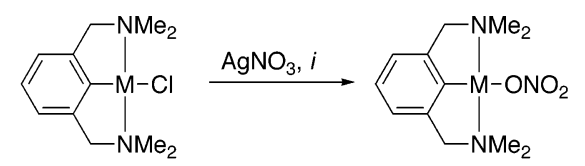

$$
M=P t(13), P d(14) \quad M=P t(11 a), P d(12 a)
$$

a (i) Acetone, RT, $16 \mathrm{~h}$.

Chart 2. Pincer Platinum(II) and Palladium(II) Complexes 11 and 12

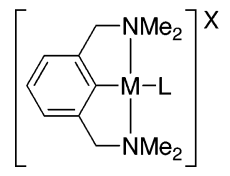

\begin{tabular}{llll}
$\mathrm{M}$ & \multicolumn{1}{c}{$\mathrm{L}$} & $\mathrm{X}$ & \\
\hline $\mathrm{Pt}$ & $\mathrm{ONO}_{2}^{-}$ & - & $\mathbf{1 1 a}$ \\
$\mathrm{Pt}$ & $\mathrm{OH}_{2}$ & $\mathrm{NO}_{3}^{-}$ & $\mathbf{1 1 b}$ \\
$\mathrm{Pt}$ & $\mathrm{OH}_{2}$ & $\mathrm{BF}_{4}^{-}$ & $\mathbf{1 1 c}$ \\
$\mathrm{Pd}$ & $\mathrm{ONO}_{2}^{-}$ & - & $\mathbf{1 2 a}$ \\
$\mathrm{Pd}$ & $\mathrm{OH}_{2}$ & $\mathrm{NO}_{3}^{-}$ & $\mathbf{1 2 b}$ \\
$\mathrm{Pd}$ & $\mathrm{OH}_{2}$ & $\mathrm{BF}_{4}^{-}$ & $\mathbf{1 2 c}$
\end{tabular}

The coordinated nitrate anions can easily be replaced by pyridyl groups in self-assembly reactions (vide infra), ${ }^{26}$ which are often carried out in aqueous solutions because of the good solubility of nitrate complexes in that environment. Although nitrate counteranions are commonly reported to be coordinated to platinum(II) and palladium(II) centers, ${ }^{26}$ it has also been reported that in water-containing solutions the nitrate ligand can be replaced by a water molecule depending on the conditions employed, e.g., the water concentration. ${ }^{27-29}$

To investigate the nature of ligand coordination in aqueous media, i.e., to answer the question of whether the complexes are neutral with formula $\left[\mathrm{M}(\mathrm{NCN})\left(\mathrm{NO}_{3}\right)\right](\mathrm{M}=\mathrm{Pt}(\mathbf{1 1 a})$, $\mathrm{Pd}(12 \mathrm{a})$; Chart 2) or cationic with formula $\left[\mathrm{M}(\mathrm{NCN})\left(\mathrm{OH}_{2}\right)\right]$ $\left(\mathrm{NO}_{3}\right)(\mathrm{M}=\mathrm{Pt}(\mathbf{1 1 b}), \mathrm{Pd}(\mathbf{1 2 b}))$, we studied a series of $\mathrm{Pt}$ and $\mathrm{Pd}$ complexes by means of X-ray diffraction and IR and Raman spectroscopy.

Crystallization of water-free complex 11a was carried out in both the absence and presence of $\mathrm{H}_{2} \mathrm{O}$. The X-ray crystal structure determination of 11a with crystals obtained by layering a concentrated solution of $\mathbf{1 1 a}$ in acetone with $\mathrm{Et}_{2} \mathrm{O}$ showed it to be the expected monomeric nitrate complex. However, layering a concentrated solution of 11a in an acetone/ $\mathrm{H}_{2} \mathrm{O}$ mixture (9:1, v/v) with $\mathrm{Et}_{2} \mathrm{O}$ afforded suitable crystals of the water adduct of 11a, $\left[\mathrm{Pt}(\mathrm{NCN})\left(\mathrm{NO}_{3}\right)\right] \cdot\left(\mathrm{H}_{2} \mathrm{O}\right)$ $\left(11 \mathrm{a} \cdot \mathrm{H}_{2} \mathrm{O}\right)$. The molecular structures are depicted in Figure 2 , and relevant bond lengths, bond angles, and torsion angles are listed in Table 3. The molecular structures reveal platinum centers that are ligated by terdentate $\eta^{3}-N, C, N$-bound NCN ligands and monodentate $\eta^{3}-O$-bound $\mathrm{NO}_{3}{ }^{-}$anions. The $\mathrm{Pt}-\mathrm{O}$ bond lengths (2.180(3) and 2.1780(17) $\AA$ for 11a and 11a $\cdot \mathrm{H}_{2} \mathrm{O}$, respectively) are comparable to values reported for platinum(II)/nitrate complexes $(2.135-2.18 \AA) .{ }^{30}$ The

(26) Kuehl, C. J.; Mayne, C. L.; Arif, A. M.; Stang, P. J. Org. lett. 2000, 2, 3727-3729.

(27) Lippert, B.; Lock, C. J. L.; Rosenberg, B.; Zvagulis, M. Inorg. Chem. $1977,16,1525-1529$

(28) Wimmer, S.; Castan, P.; Wimmer, F. L.; Johnson, N. P. J. Chem. Soc., Dalton Trans. 1989, 403-412 and references cited therein

(29) See the review: Vincente, J.; Arcas, A. Coord. Chem. Rev. 2005, 249, 1135-1154 and references cited therein.

(30) (a) Gorla, F.; Togni, A.; Venanzi, L. M.; Albinati, A.; Lianza, F. Organometallics 1994, 13, 1607-1616. (b) Hollis, L. S.; Lippard, S. J. J. Am. Chem. Soc. 1981, 103, 6761-6763. (c) Kampf, G.; Willermann, M.; Freisinger, E.; Lippert, B. Inorg. Chim. Acta 2002, $330,179-188$ puckered five-membered chelate ring conformations, bond lengths, and bond angles of the $\mathrm{Pt}-\mathrm{NCN}$ moieties fall in the range normally observed for square-planar platinum(II) complexes containing this type of ligand. ${ }^{25,31}$ In the solid state, $\mathbf{1 1 a} \cdot \mathrm{H}_{2} \mathrm{O}$ is self-assembled into centrosymmetric dimers by hydrogen bonding between the $\mathrm{H}_{2} \mathrm{O}$ molecules as the donor and the $\mathrm{O}$ atom from the nitrate group as the acceptor; i.e., it is the $\mathrm{H}_{2} \mathrm{O}$ molecule that forms the bridges between two neutral $\left[\mathrm{Pt}(\mathrm{NCN})\left(\mathrm{NO}_{3}\right)\right]$ entities. The interatomic distances between $\mathrm{H} 1 \mathrm{O} \cdots \mathrm{O} 3$ and $\mathrm{H} 2 \mathrm{O} \cdots \mathrm{O} 1$ are 2.20(4) and 2.12(5) $\AA$, respectively, and the contact angles between $\mathrm{O} 4-$ $\mathrm{H} 1 \mathrm{O} \cdots \mathrm{O} 3$ and $\mathrm{O} 4-\mathrm{H} 2 \mathrm{O} \cdots \mathrm{O} 1$ are both $158(4)^{\circ}$. Similar assemblies of cationic platinum and palladium complexes have been reported before. ${ }^{29}$ However, in most cases the anions were bridging two cationic aqua complexes via hydrogen bonding. For example, for pincer platinum and palladium complexes, this resulted in the formation of a dimeric structure in the case of $\left[\mathrm{Pt}(\mathrm{NCN})\left(\mathrm{H}_{2} \mathrm{O}\right)\right]\left(\mathrm{OSO}_{2} \mathrm{CF}_{3}\right)^{31 \mathrm{~b}}$ (15) and a polymeric structure for $\left[\mathrm{Pd}(\mathrm{NCN})\left(\mathrm{H}_{2} \mathrm{O}\right)\right]\left(\mathrm{BF}_{4}\right)$ $(\mathbf{1 2 c})^{32}$ in the solid state (Chart 3).

Similar to 3a, the crystal structure of 11a has short intermolecular contacts between the platinum center and the benzylic hydrogen atoms $\mathrm{H} 7 \mathrm{~A}$ and $\mathrm{H} 10 \mathrm{~A}$, respectively, with distances of 2.96 and $2.90 \AA$, which are shorter than the sum of their contact radii.

Raman and IR spectra of nitrate complexes have been widely used to gain information about the mode of coordination of the nitrate anion and the covalent character of the $\mathrm{O}-\mathrm{M}$ bond. The planar symmetric free nitrate ion $\left(\mathrm{NO}_{3}{ }^{-}\right)$ has four fundamental frequencies of absorption according to its $D_{3 h}$ symmetry. ${ }^{33-35}$ However, when monodentate coordinated to a metal center, the symmetry of the nitrate is lowered to $C_{2 v}$ or $C_{s}$ (as we observed) and the $\mathrm{N}-\mathrm{O}(\mathrm{M})$ bond order is also reduced. As a result, variations on the modes and frequencies of vibration of the nitrate ligand will be observed. For instance, the degenerated modes $\left(E^{\prime}\right)$ of the free nitrate anion will split into the asymmetric and symmetric modes upon coordination. ${ }^{36-39}$

Solid-state IR spectra of complexes 11c and 12c with noncoordinating $\mathrm{BF}_{4}^{-}$anions, which were used as models

(31) (a) Amijs, C. H. M.; Kleij, A. W.; van Klink, G. P. M.; Spek, A. L.; van Koten, G. Organometallics 2005, 24, 2773-2779. (b) Schmülling, M.; Grove, D. M.; van Koten, G.; van Eldik, R.; Veldman, N.; Spek, A. L. Organometallics 1996, 15, 1384-1391.

(32) van den Broeke, J.; Heeringa, J. J. H.; Chuchuryukin, A. V.; Kooijman, H.; Mills, A. M.; Spek, A. L.; van Lenthe, J. H.; Ruttink, P. J. A.; Deelman, B.-J.; van Koten, G. Organometallics 2004, 23, 2287-2294.

(33) Nakamoto, K. Infrared and Raman Spectra of Inorganic and Coordination Compounds, 4th ed.; Wiley-Interscience: New York, 1986.

(34) The four active frequencies in IR for the free $\mathrm{NO}_{3}{ }^{-}$ion with $D_{3 h}$ symmetry are $v_{1}\left(\mathrm{~A}_{1}^{\prime}\right),{ }^{35} v_{2}\left(\mathrm{~A}^{\prime \prime}{ }_{2}\right), v_{3}\left(\mathrm{E}^{\prime}\right)$, and $v_{4}\left(\mathrm{E}^{\prime}\right)$.

(35) Although the mode $v_{1}\left(\mathrm{~A}_{1}^{\prime}\right)$ (NO stretching) is expected to be only active in Raman, it has been shown experimentally that this mode can be weakly IR-active because of the deformation of the nitrate ion in the molecular field of the crystals. See: Mizushima, S. I.; Quagliano, J. V. J. Am. Chem. Soc. 1953, 75, 4870.

(36) For monodentate-coordinated nitrates with $C_{s}$ symmetry, the active frequencies in both Raman and IR are $v_{1}\left(\mathrm{~A}^{\prime}\right), v_{2}\left(\mathrm{~A}^{\prime}\right), v_{3}\left(\mathrm{~A}^{\prime}\right), v_{4}\left(\mathrm{~A}^{\prime}\right)$, $v_{5}\left(\mathrm{~A}^{\prime}\right)$, and $v_{6}\left(\mathrm{~A}^{\prime \prime}\right)$.

(37) (a) Brintzinger, H.; Hester, R. E. Inorg. Chem. 1966, 5, 980-985. (b) Hester, R. E.; Grossman, W. E. L. Inorg. Chem. 1966, 5, 1308-1312.

(38) (a) Morris, V. R.; Bhatia, S. C.; Hall, J. H., Jr. J. Phys. Chem. 1991, 95, 9203-9208. (b) McLaughlin, R. P.; Bird, B.; Reid, P. J. Spectrochim. Acta, Part A 2002, 58, 2571-2580. 

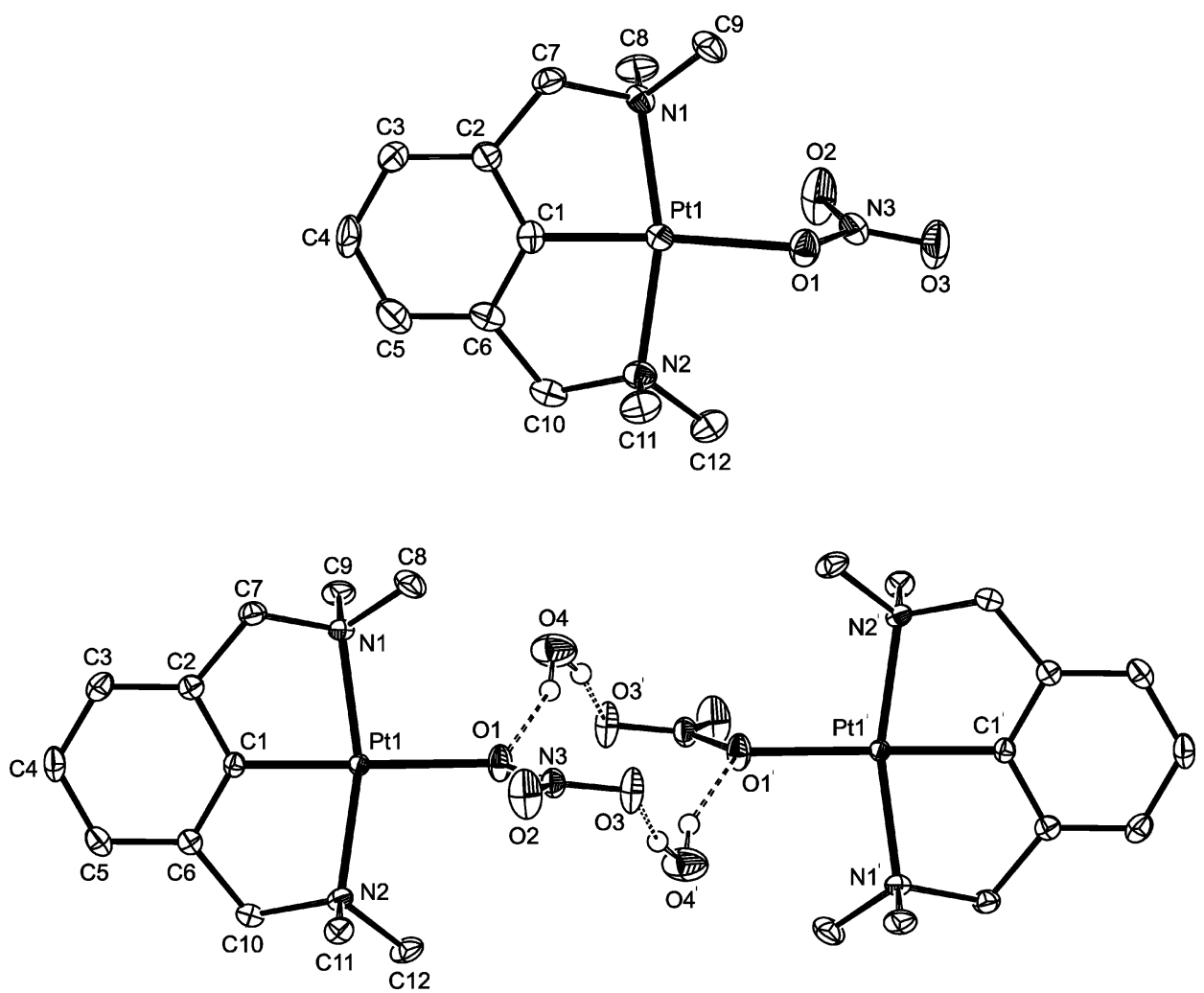

Figure 2. Displacement ellipsoid plot (50\% probability level) of the molecular structure of 11a (top) and a dimeric $\mathbf{1 1} \cdot \mathrm{H}_{2} \mathrm{O}$ (bottom), with the adopted labeling schemes. Hydrogen atoms (except for the water molecule) have been omitted for clarity (symmetry operation $i$ : $1-x, 1-y, 1-z$ ). Note that the $\mathrm{H}_{2} \mathrm{O}$ molecules represent the bridges between the neutral $\left[\mathrm{Pt}(\mathrm{NCN})\left(\mathrm{NO}_{3}\right)\right]$ entities.

Table 3. Selected Bond Lengths (A), Bond Angles (deg), and Torsion Angles (deg) for 11a and 11a $\cdot \mathrm{H}_{2} \mathrm{O}$

\begin{tabular}{|c|c|c|}
\hline & $11 a$ & $\mathbf{1 1 a} \cdot \mathrm{H}_{2} \mathrm{O}$ \\
\hline \multicolumn{3}{|c|}{ Interatomic Distances } \\
\hline $\mathrm{Pt} 1-\mathrm{N} 1$ & $2.089(4)$ & $2.0847(18)$ \\
\hline $\mathrm{Pt} 1-\mathrm{N} 2$ & $2.087(4)$ & $2.0847(18)$ \\
\hline $\mathrm{Pt} 1-\mathrm{C} 1$ & $1.923(3)$ & $1.908(2)$ \\
\hline $\mathrm{Pt} 1-\mathrm{O} 1$ & $2.180(3)$ & $2.1780(17)$ \\
\hline $\mathrm{O} 4-\mathrm{H} 1 \mathrm{O}, \mathrm{O} 4-\mathrm{H} 2 \mathrm{O}$ & & $0.81(4), 0.81(5)$ \\
\hline $\mathrm{H} 1 \mathrm{O} \cdots \mathrm{O}^{i}{ }^{i}, \mathrm{H} 2 \mathrm{O} \cdots \mathrm{O} 1$ & & $2.20(4), 2.12(5)$ \\
\hline $\mathrm{O} 4 \cdots \cdot \mathrm{O}^{i}, \mathrm{O} 4 \cdots \mathrm{O} 1$ & & $2.972(3), 2.884(3)$ \\
\hline \multicolumn{3}{|c|}{ Interatomic Angles and Torsion Angles } \\
\hline $\mathrm{N} 1-\mathrm{Pt} 1-\mathrm{N} 2$ & $163.82(14)$ & $164.25(8)$ \\
\hline $\mathrm{C} 1-\mathrm{Pt} 1-\mathrm{O} 1$ & $173.0(4)$ & $173.58(7)$ \\
\hline $\mathrm{N} 1-\mathrm{Pt} 1-\mathrm{C} 1$ & $81.9(4)$ & $82.35(9)$ \\
\hline $\mathrm{N} 2-\mathrm{Pt} 1-\mathrm{C} 1$ & $82.0(4)$ & $81.95(9)$ \\
\hline $\mathrm{O} 4-\mathrm{H} 1 \mathrm{O} \cdots \mathrm{O} 3, \mathrm{O} 4-\mathrm{H} 2 \mathrm{O} \cdots \mathrm{O} 1$ & & $158(4), 158(4)$ \\
\hline $\mathrm{N} 1-\mathrm{Pt} 1-\mathrm{C} 1-\mathrm{C} 2$ & $-13.4(7)$ & $-11.19(18)$ \\
\hline $\mathrm{N} 2-\mathrm{Pt} 1-\mathrm{C} 1-\mathrm{C} 6$ & $-12.4(7)$ & $-14.65(18)$ \\
\hline
\end{tabular}

of aqua-coordinated compounds, showed the typical intense absorption of $\mathrm{H}_{2} \mathrm{O}$ at 3428 and $3468 \mathrm{~cm}^{-1}$, respectively (Table 4). Complexes 11a and 12a, which were synthesized in technical acetone, did not show any significant $\mathrm{H}_{2} \mathrm{O}$ absorptions, and the absorptions of coordinated nitrate ligands were unambiguously identified in the IR spectra. In general, the symmetric stretching band $v_{1}\left(\mathrm{~A}_{1}^{\prime}\right)$ in the free $\mathrm{NO}_{3}{ }^{-}$ion appears around $1050 \mathrm{~cm}^{-1}$, while for a purely covalent (N)O-C bond $\left(\mathrm{O}_{2} \mathrm{NOCH}_{3}\right)$, this stretching band is observed at $854 \mathrm{~cm}^{-1} \cdot{ }^{40}$ The energy difference $\Delta$ between the

(39) (a) Gatehouse, B. M.; Livingstone, S. E.; Nyholm, R. S. J. Chem. Soc. 1957, 4222-4225. (b) Gatehouse, B. M.; Livingstone, S. E.; Nyholm, R. S. J. Inorg. Nucl. Chem. 1958, 8, 75-78. (c) Ferraro, J. R. J. Mol. Spectrosc. 1960, 4, 99-105. asymmetric and symmetric stretching modes $v_{1}\left(\mathrm{~A}^{\prime}\right)$ and $v_{2}\left(\mathrm{~A}^{\prime}\right)$ (in $C_{s}$ symmetry), respectively, of the same compound, which appears for $\eta^{1}$-coordinated nitrate compounds as a result of the loss of degeneracy of the $v_{3}\left(\mathrm{E}^{\prime}\right)$ mode of the nitrate ion, has also been used to measure the covalent character of the (N)O-M bond. ${ }^{33,41}$ For example, the $\Delta$ value of nitromethane is $385 \mathrm{~cm}^{-1}$, while for the free ion, it is zero. The observed energy differences $\Delta\left(\nu_{1}\left(\mathrm{~A}^{\prime}\right)-v_{2}\left(\mathrm{~A}^{\prime}\right)\right)$ and $\left(v_{1}\left(\mathrm{~A}_{1}^{\prime}\right)-v_{3}\left(\mathrm{~A}^{\prime}\right)\right)$ between the symmetric stretching band of the free $\mathrm{NO}_{3}{ }^{-}$ion and the $\mathrm{N}-\mathrm{O}(\mathrm{M})$ stretching of the coordinated nitrate ligand are listed in Table 4.

The split between the asymmetric and symmetric stretching modes $v_{1}\left(\mathrm{~A}^{\prime}\right)$ and $v_{2}\left(\mathrm{~A}^{\prime}\right)$ is larger in 11a than in 12a. The same trend is observed for the difference $v_{1}\left(\mathrm{~A}_{1}^{\prime}\right)-v_{3}\left(\mathrm{~A}^{\prime}\right)$, indicating a more covalent character of the $(\mathrm{N}) \mathrm{O}-\mathrm{Pt}$ bond in comparison to $(\mathrm{N}) \mathrm{O}-\mathrm{Pd}$.

The IR spectrum of crystals of the water adduct complex $11 a \cdot \mathrm{H}_{2} \mathrm{O}$ showed only a small variation for the bands of the nitrate ligand in comparison to the water-free analogue 11a, indicating $\mathrm{NO}_{3}$ coordination to the metal. Two well-defined bands, for the asymmetric and symmetric $\left(v_{3}\right.$ and $\left.v_{1}\right) \mathrm{O}-\mathrm{H}$ stretching modes, were observed for the water molecule. A thin weak absorption at $1613 \mathrm{~cm}^{-1}$ was also detected, which is characteristic for the bending mode of water in the crystal lattice. The appearance of these fine resolved bands can be the result of interactions of the water molecule through

(40) Brand, J. C. D.; Cawthon, T. M. J. Am. Chem. Soc. 1955, 77, 319323.

(41) (a) Tessier, C.; Rochon, F. D. Inorg. Chim. Acta 2001, 322, 37-46. (b) Rochon, F. D.; Buculei, V. Can. J. Chem. 2004, 82, 524-532. 
Chart 3. Hydrogen-Bonded Assemblies of $\mathbf{1 5}$ and $\mathbf{1 2 c}$ in the Solid State ${ }^{a, 31 b, 32}$
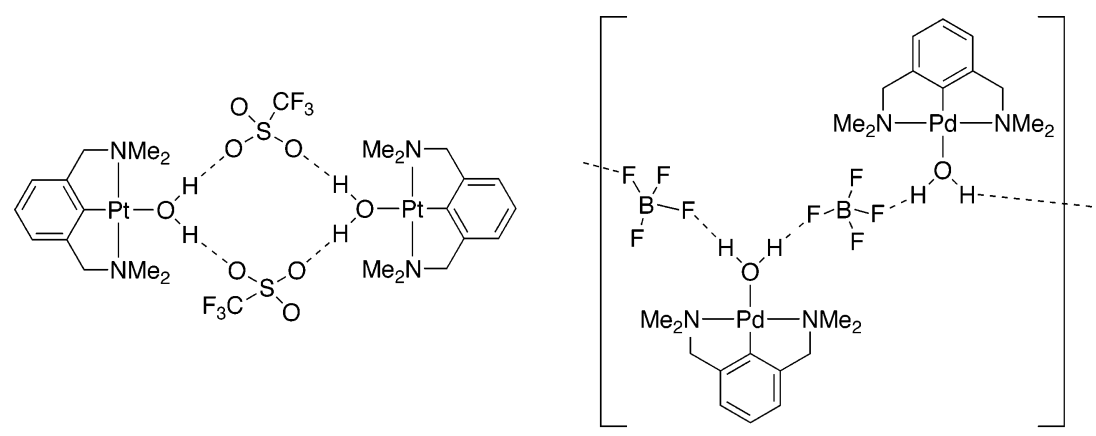

15

$12 c$

${ }^{a}$ Note that the anions form the bridges between the $\left[\mathrm{M}(\mathrm{NCN})\left(\mathrm{H}_{2} \mathrm{O}\right)\right]^{+}$cations.

Table 4. Selected IR Bands $\left(\mathrm{cm}^{-1}\right)^{a}$ of $\mathrm{NCN}-\mathrm{Pt}^{\mathrm{II}}$ and $-\mathrm{Pd}^{\mathrm{II}}$ Complexes ${ }^{33}$

\begin{tabular}{|c|c|c|c|c|c|c|}
\hline complex & $v\left(\mathrm{H}_{2} \mathrm{O}\right)\left(v_{3}\right)_{\mathrm{as}},\left(v_{1}\right)_{\mathrm{ss}},\left(v_{2}\right)_{\mathrm{b}}$ & $\begin{array}{l}v_{1}\left(\mathrm{~A}^{\prime}\right) \\
\left(\mathrm{NO}_{2}\right)_{\mathrm{as}}\end{array}$ & $\begin{array}{l}v_{2}\left(\mathrm{~A}^{\prime}\right) \\
\left(\mathrm{NO}_{2}\right)_{\mathrm{ss}}\end{array}$ & $\begin{array}{c}v_{3}\left(\mathrm{~A}^{\prime}\right) \\
(\mathrm{N}-\mathrm{OM})_{\mathrm{s}}\end{array}$ & $\Delta\left(v_{1}\left(\mathrm{~A}^{\prime}\right)-v_{2}\left(\mathrm{~A}^{\prime}\right)\right)$ & $\begin{array}{c}v_{1}\left(\mathrm{~A}_{1}^{\prime}\right)_{\mathrm{ss}}\left(\text { free } \mathrm{NO}_{3}\right)^{b}- \\
v_{3}\left(\mathrm{~A}^{\prime}\right)_{\mathrm{s}}\left(\eta^{1}-\mathrm{ON}\right)\end{array}$ \\
\hline $11 \mathbf{a}^{c}$ & & $1417_{\mathrm{s}}$ & $1281_{\mathrm{vs}}$ & $1014_{\mathrm{m}}$ & 136 & 36 \\
\hline 12a & & $1411_{\mathrm{s}}$ & $1289_{\mathrm{vs}}$ & $1027_{\mathrm{m}}$ & 122 & 23 \\
\hline $\mathbf{1 1 a} \cdot \mathrm{H}_{2} \mathrm{O}^{c}$ & $3573_{\mathrm{s}}, 3494_{\mathrm{m}}, 1613_{\mathrm{w}}$ & $1417_{\mathrm{m}}$ & $1286_{\mathrm{vs}}$ & $1008_{\mathrm{m}}$ & 131 & 42 \\
\hline $11 \mathrm{c}$ & $3428_{\mathrm{vs}(v 3+v 1)}$ & & & & & \\
\hline $12 \mathrm{c}$ & $3468_{\operatorname{br}(v 3+v 1)}$ & & & & & \\
\hline
\end{tabular}

${ }^{a}$ Solid state. IR intensities: $\mathrm{vs}=$ very strong, $\mathrm{s}=$ strong, $\mathrm{m}=$ medium, $\mathrm{w}=$ weak, $\mathrm{br}=$ broad. Type of vibration: as $=$ asymmetric stretching, $\mathrm{ss}=$ symmetric stretching, $\mathrm{s}=$ stretching, $\mathrm{b}=$ bending. ${ }^{b} v_{1}\left(\mathrm{~A}_{1}^{\prime}\right)=1050 \mathrm{~cm}^{-1}$ for the free $\mathrm{NO}_{3}{ }^{-}$ion. ${ }^{c}$ Samples measured from crystals obtained for $\mathrm{X}$-ray spectroscopy.

hydrogen bonding with the nitrate ligand. ${ }^{33}$ In contrast, for compounds 11c and 12c with water coordinated to the metal center, the stretching vibrations $v_{3}$ and $v_{1}$ appear as one broad signal.

Attempts to synthesize and isolate the cationic aqua complexes $\mathbf{1 1 b}$ and $\mathbf{1 2 b}$ in aqueous solution were carried out by dissolving complexes 11a and 12a in water and stirring for $16 \mathrm{~h}$, followed by freeze-drying of the reaction mixtures. The IR spectra of the products pointed to coordination of an $\mathrm{NO}_{3}{ }^{-}$anion to the metal center. A weak band at $3571 \mathrm{~cm}^{-1}$ corresponding to the water molecule was also observed but could not unambiguously be determined as being from an aqua complex or free water.

Raman analysis was also carried out for complexes 11a, $\mathbf{1 1 b}, \mathbf{1 2} \mathbf{a}$, and the crystals of $\mathbf{1 1 a} \cdot \mathrm{H}_{2} \mathrm{O}$, both in the solid state and in aqueous solution to verify the presence of free $\mathrm{NO}_{3}{ }^{-}$ anions. For all platinum compounds $\mathbf{1 1 a}, \mathbf{1 1} \mathbf{a} \cdot \mathrm{H}_{2} \mathrm{O}$, and $\mathbf{1 1 b}$, two bands were observed in the solid state at $\sim 1039$ and $\sim 1015 \mathrm{~cm}^{-1}$, which correspond to free and coordinated $\mathrm{NO}_{3}{ }^{-}$ anions, $v_{1}\left(\mathrm{~A}_{1}^{\prime}\right)$ and $v_{3}\left(\mathrm{~A}^{\prime}\right)$, respectively. The peaks appeared in similar ratios with respect to each other $(\sim 1: 1)$. For the palladium complex 12a, analogous peaks at 1038 and 1025 $\mathrm{cm}^{-1}$ were observed. On the other hand, Raman spectra of aqueous solutions of the complexes 11a, 11a $\cdot \mathrm{H}_{2} \mathrm{O}, \mathbf{1 1 b}$, and 12a showed only a strong signal at $\sim 1046 \mathrm{~cm}^{-1}$, which is indicative of the presence of only noncoordinated nitrate anions.

IR measurements pointed toward the existence of pure $\left[\mathrm{M}(\mathrm{NCN})\left(\mathrm{NO}_{3}\right)\right]$ complexes 11a and 12a in the solid state because no significant signals for water were observed for 11a and 12a. The presence of aqua complexes [M(NCN)$\left.\left(\mathrm{H}_{2} \mathrm{O}\right)\right]\left(\mathrm{NO}_{3}\right)$ in the samples of 11a and 12a was not observed by IR because of the fact that the symmetric NO stretching $\left(v_{1}\left(\mathrm{~A}^{\prime}{ }_{1}\right)\right)$ of free nitrate is not IR-active. ${ }^{35}$ The other vibration modes $v_{3}\left(\mathrm{E}^{\prime}\right), v_{2}\left(\mathrm{~A}^{\prime \prime}{ }_{2}\right)$, and $v_{4}\left(\mathrm{E}^{\prime}\right)$, which are active, were not observed because of overlap. On the contrary, from the Raman analysis, it can be concluded that both [M(NCN)$\left.\left(\mathrm{NO}_{3}\right)\right]$ and $\left[\mathrm{M}(\mathrm{NCN})\left(\mathrm{H}_{2} \mathrm{O}\right)\right]\left(\mathrm{NO}_{3}\right)$ complexes were present in the samples of 11a and 12a because the signals of both free and coordinated nitrate were observed. It is likely that a dynamic exchange process between nitrate-to-metal (11a and 12a) and water-to-metal (11b and 12b) coordination exists during the synthesis or isolation of the complexes. This was also demonstrated by the Raman analysis in aqueous solutions, in which only complexes 11b and 12b were observed, indicating a complete shift of the equilibrium to water-to-metal coordination. This behavior was also observed for dissolution of $\left[\mathrm{PdL}\left(\mathrm{NO}_{3}\right)_{2}\right](\mathrm{L}=1$-methyl-2,2'-bipyridin3-ylium) in water. ${ }^{42}$

It should be noted that the absence of coordinated water in the crystals of $\mathbf{1 1 a}$ and $\mathbf{1 1 a} \cdot \mathrm{H}_{2} \mathrm{O}$, measured with X-ray crystallographic studies, does not exclude the presence of the aqua complex $\mathbf{1 1 b}$. Attempts to crystallize complexes 11b and 12b from aqueous solutions were without success. These results indicate that only IR analysis and/or X-ray crystallography are not sufficient to confirm the presence or absence of water in these kinds of complexes, although conclusions have been drawn from results obtained by these techniques. ${ }^{29,42,43}$ By Raman analysis, however, noncoordinated nitrate groups can be easily observed in the sample, providing definite proof for the presence of (non)coordinating nitrate anions.

Coordination Reactions with $\left[\mathrm{M}(\mathbf{N C N})\left(\mathbf{N O}_{3}\right)\right]$. The coordination reactions between solutions of $\mathbf{3 a}, \mathbf{b}$ and 2 equiv

(42) Castan, P.; Jaud, J.; Wimmer, S.; Wimmer, F. L. J. Chem. Soc., Dalton Trans. 1991, 1155-1158.

(43) Anandhi, U.; Holbert, T.; Lueng, D.; Sharp, P. R. Inorg. Chem. 2003, 42, 1282-1295. 
Scheme 4. Coordination Reactions of $\mathbf{3 a}$ and $\mathbf{3 b}$ with Cationic Platinum(II) and Palladium(II) Pincer Nitrate Complexes ${ }^{a}$

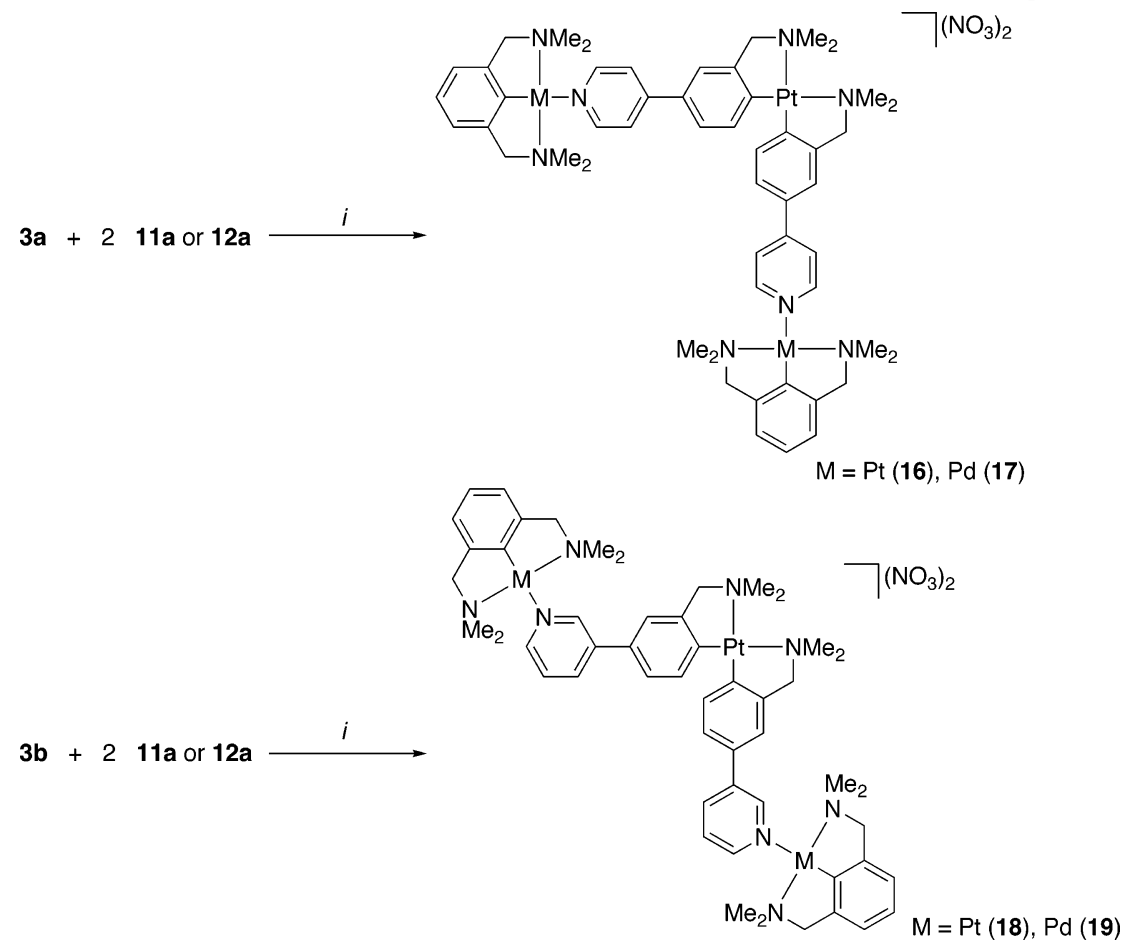

${ }^{a}$ (i) Acetone, $\mathrm{H}_{2} \mathrm{O}, \mathrm{RT}, 30 \mathrm{~min}$.

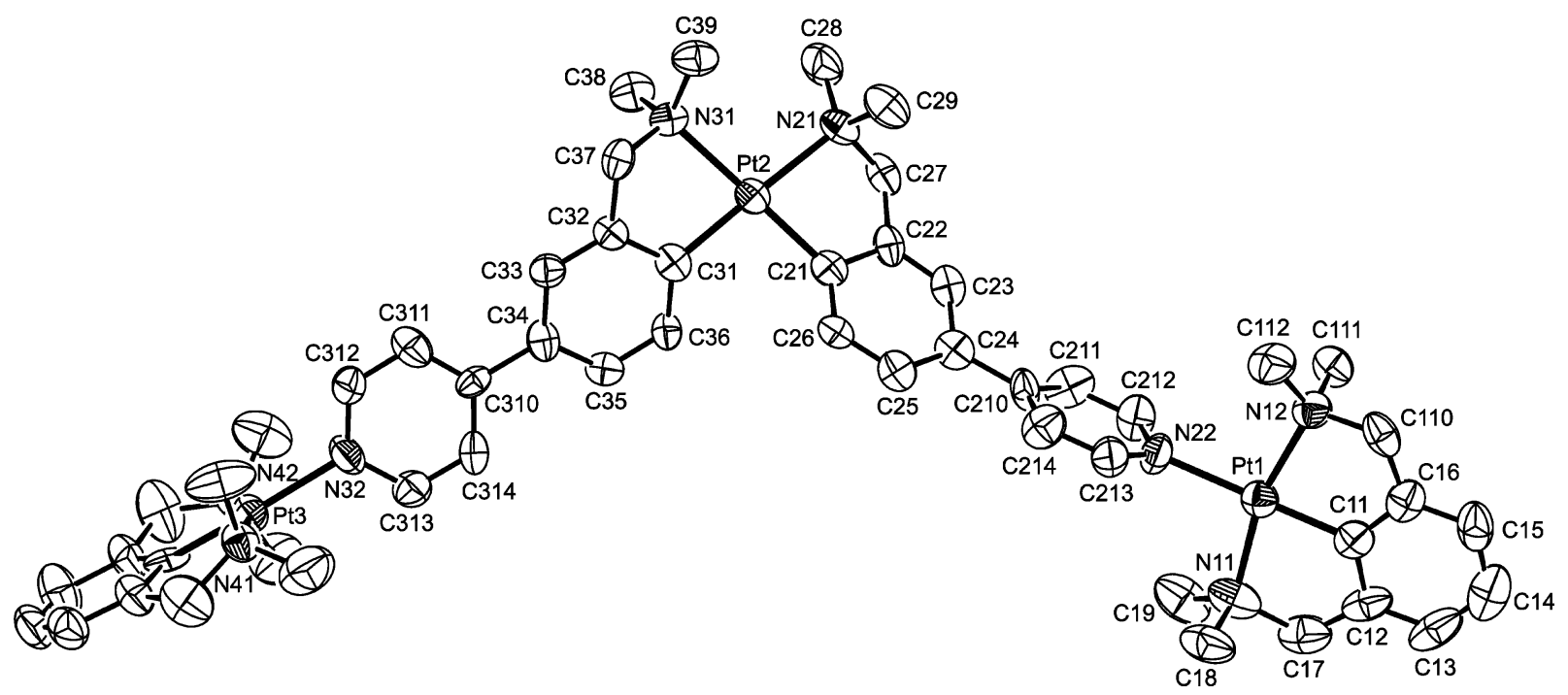

Figure 3. Displacement ellipsoid plot (50\% probability level) of the molecular structure of the dication of trisplatinum complex 16, with the adopted labeling scheme. Hydrogen atoms, disordered solvent molecules, and nitrate anions have been omitted for clarity.

of 11a or 12a in deuterated solvents (Scheme 4) were performed by stirring the mixtures for $30 \mathrm{~min}$ at room temperature, after which ${ }^{1} \mathrm{H}$ NMR spectra were recorded. The spectra showed in each reaction the formation of a single compound, i.e., corner complexes coordinated with the pyridyl groups to two metal pincer entities. The thus-formed dicationic trinuclear complexes 16-19 were isolated as lightyellow solids by slow precipitation with $\mathrm{Et}_{2} \mathrm{O}$.

Single crystals of complex $\mathbf{1 6}$ suitable for an X-ray crystal structure determination were obtained by slow diffusion of $\mathrm{Et}_{2} \mathrm{O}$ into a concentrated acetone $/ \mathrm{H}_{2} \mathrm{O}(2: 1)$ mixture. The molecular structure is depicted in Figure 3, and relevant bond lengths, bond angles, and torsion angles are listed in Table
5. The molecular structure of $\mathbf{1 6}$ shows the same cis conformation of the central moiety as was present in $\mathbf{3 a}$, with similar angles and distances. To both pyridyl groups is coordinated the NCN-platinum(II) complex in a trans fashion $\left(179.1(3)^{\circ} \mathrm{N} 22-\mathrm{Pt} 1-\mathrm{C} 11\right.$ and $\left.177.2(3)^{\circ} \mathrm{N} 32-\mathrm{Pt} 3-\mathrm{C} 41\right)$. The pincer platinum moieties are placed nearly perpendicular to the pyridyl planes, with torsion angles of $91.1(7)^{\circ}$ and $83.6(7)^{\circ}$ for $\mathrm{N} 11-\mathrm{Pt} 1-\mathrm{N} 22-\mathrm{C} 213$ and $\mathrm{N} 41-\mathrm{Pt} 3-\mathrm{N} 32-$ $\mathrm{C} 313$, respectively. These values are consistent with those observed for related NCN-Pt-pyridyl complexes. ${ }^{10}$ The PtN(pyridyl) distances (2.141(6) A for both Pt1-N22 and Pt3N32) agree with those observed for trans-pyridyl-coordinated $\mathrm{N} \wedge \mathrm{C} \wedge \mathrm{N}$ ligands $\left(\left[\mathrm{Pt}\left(\mathrm{pip}_{2} \mathrm{NCN}\right)(\mathrm{py})\right]^{+}(2.138-2.159 \AA)^{21 b, \mathrm{~d}}\right.$ 
Table 5. Selected Bond Lengths ( $\AA$ ), Bond Angles (deg), and Torsion Angles (deg) for $\mathbf{1 6}$

\begin{tabular}{|c|c|c|c|}
\hline \multicolumn{4}{|c|}{ Interatomic Distances } \\
\hline $\mathrm{Pt} 2-\mathrm{N} 21$ & $2.197(7)$ & Pt2-N31 & $2.203(8)$ \\
\hline $\mathrm{Pt} 2-\mathrm{C} 21$ & $1.997(12)$ & Pt2-C31 & $1.973(7)$ \\
\hline Pt1-N11 & $2.073(7)$ & $\mathrm{Pt} 3-\mathrm{N} 41$ & $2.074(7)$ \\
\hline $\mathrm{Pt} 1-\mathrm{N} 12$ & $2.072(7)$ & $\mathrm{Pt} 3-\mathrm{N} 42$ & $2.073(7)$ \\
\hline Pt1-N22 & $2.141(6)$ & $\mathrm{Pt} 1-\mathrm{C} 11$ & $1.942(11)$ \\
\hline $\mathrm{Pt} 3-\mathrm{N} 32$ & $2.141(6)$ & $\mathrm{Pt} 3-\mathrm{C} 41$ & $1.820(9)$ \\
\hline \multicolumn{4}{|c|}{ Interatomic Angles and Torsion Angles } \\
\hline $\mathrm{N} 21-\mathrm{Pt} 2-\mathrm{N} 31$ & 102.1(3) & $\mathrm{C} 21-\mathrm{Pt} 2-\mathrm{C} 31$ & $97.2(4)$ \\
\hline $\mathrm{N} 21-\mathrm{Pt} 2-\mathrm{C} 31$ & $170.4(3), 171.2(3)$ & $\mathrm{N} 21-\mathrm{Pt} 2-\mathrm{C} 21$ & $80.9(4)$ \\
\hline $\mathrm{N} 31-\mathrm{Pt} 2-\mathrm{C} 21$ & & $\mathrm{~N} 31-\mathrm{Pt} 2-\mathrm{C} 31$ & $81.2(3)$ \\
\hline $\mathrm{N} 11-\mathrm{Pt} 1-\mathrm{N} 12$ & $164.8(4)$ & $\mathrm{N} 22-\mathrm{Pt} 1-\mathrm{C} 11$ & $179.2(3)$ \\
\hline $\mathrm{N} 41-\mathrm{Pt} 3-\mathrm{N} 42$ & $164.4(3)$ & N32-Pt3-C41 & $177.2(4)$ \\
\hline $\mathrm{N} 11-\mathrm{Pt} 1-\mathrm{C} 11$ & $82.9(4)$ & N41-Pt3-C41 & $83.5(3)$ \\
\hline $\mathrm{N} 12-\mathrm{Pt} 1-\mathrm{C} 11$ & $82.0(4)$ & $\mathrm{N} 42-\mathrm{Pt} 3-\mathrm{C} 41$ & $80.9(3)$ \\
\hline $\mathrm{C} 21-\mathrm{Pt} 2-\mathrm{C} 31-\mathrm{C} 36$ & $-31.0(8)$ & $\mathrm{C} 31-\mathrm{Pt} 2-\mathrm{C} 21-\mathrm{C} 26$ & $-36.2(8)$ \\
\hline $\mathrm{C} 211-\mathrm{C} 210-\mathrm{C} 24-\mathrm{C} 23$ & $-29.4(15)$ & C311-C310-C34-C33 & $23.5(11)$ \\
\hline $\mathrm{N} 11-\mathrm{Pt} 1-\mathrm{C} 11-\mathrm{C} 12$ & $11.1(7)$ & $\mathrm{N} 41-\mathrm{Pt} 3-\mathrm{C} 41-\mathrm{C} 42$ & $-2.8(6)$ \\
\hline $\mathrm{N} 12-\mathrm{Pt} 1-\mathrm{C} 11-\mathrm{C} 16$ & $9.3(7)$ & $\mathrm{N} 42-\mathrm{Pt} 3-\mathrm{C} 41-\mathrm{C} 46$ & $-11.3(7)$ \\
\hline $\mathrm{N} 11-\mathrm{Pt} 1-\mathrm{N} 22-\mathrm{C} 213$ & $91.1(7)$ & $\mathrm{N} 41-\mathrm{Pt} 3-\mathrm{N} 32-\mathrm{C} 313$ & $83.6(7)$ \\
\hline
\end{tabular}

Table 6. Selected ${ }^{1} \mathrm{H}$ NMR Data for Complexes $\mathbf{3}$ and $\mathbf{1 6}-\mathbf{2} \mathbf{1}^{a}$

\begin{tabular}{|c|c|c|c|c|c|c|}
\hline \multirow[b]{2}{*}{ complex } & \multicolumn{2}{|c|}{$\mathrm{NMe}_{2}\left({ }^{3} J_{\mathrm{Pt}-\mathrm{H}}\right)$} & \multicolumn{2}{|c|}{$\mathrm{CH}_{2} \mathrm{~N}\left({ }^{3} J_{\mathrm{Pt}-\mathrm{H}}\right)$} & \multicolumn{2}{|c|}{ pyridyl protons } \\
\hline & $\eta^{2}-\mathrm{CN}$ & $\eta^{3}-\mathrm{NCN}$ & $\eta^{2}-\mathrm{CN}$ & $\eta^{3}-\mathrm{NCN}$ & $\alpha$ & $\beta$ \\
\hline $3 \mathbf{a}^{b}$ & $2.86(11)$ & & $3.99(16)$ & & $8.59(\mathrm{~d})$ & $7.58(\mathrm{~d})$ \\
\hline $3 \mathbf{b}^{b}$ & 2.84 & & 3.96 & & $8.85,8.48$ (d) & 7.87 \\
\hline $16^{c}$ & 2.88 & 2.79 & 3.95 & 4.18 & $8.91(\mathrm{~d})$ & $8.01(\mathrm{~d})$ \\
\hline $17^{c}$ & 2.78 & 2.64 & 3.93 & 4.04 & $8.81(\mathrm{bs})$ & 7.87 (bs) \\
\hline $18^{c}$ & 2.79 & 2.82 & 3.93 & 4.20 & $9.21,8.91(\mathrm{~d})$ & $8.29(\mathrm{~d})$ \\
\hline $19^{c}$ & 2.77 & 2.71 & 3.90 & 4.05 & $9.09(\mathrm{bs}), 8.74(\mathrm{bs})$ & 8.17 (bs) \\
\hline $\mathbf{2 0}^{c}$ & 2.79 & 2.79 & $3.92,3.93$ & 4.18 & $8.90(\mathrm{~d}), 8.46(\mathrm{~d})$ & 8.00 (d), 7.59 (d) \\
\hline $21^{c}$ & 2.78 & 2.67 & 3.92 & 4.08 & 8.74 (bs) & 7.80 (bs) \\
\hline
\end{tabular}

${ }^{a}$ Chemical shifts in ppm, coupling in hertz, singlet resonances unless stated otherwise, doublet (d), broad singlet (bs), $25{ }^{\circ} \mathrm{C} .{ }^{b}$ In $\mathrm{CD}_{2} \mathrm{Cl}_{2}$ solutions. ${ }^{c}$ In acetone- $d_{6} / \mathrm{D}_{2} \mathrm{O}(2: 1)$ solutions.

and $[\mathrm{Pt}(\mathrm{NCN})(\mathrm{py})]^{+}(2.183-2.192 \AA) .{ }^{10,21 \mathrm{c}}$ The puckered five-membered chelate ring conformations, bond lengths, and bond angles of the $\mathrm{Pt}-\mathrm{NCN}$ moieties fall in the range normally observed for square-planar platinum(II) complexes containing this type of ligand. ${ }^{25,31}$

Although both complexes $\mathbf{3 a}$ and $\mathbf{1 6}$ have distorted squareplanar geometries with torsion angles between $29.8(4)^{\circ}$ and $36.2(7)^{\circ}$ (Figure 4 ) for the aryl rings around the platinum center, this should not hamper the possible formation of closed and flat molecular structures such as molecular squares. These complexes have proven to be very flexible in solution because twisting of the arene rings with respect to each other was even observed at low temperatures (vide infra). ${ }^{6}$

Structure in Solution. The ${ }^{1} \mathrm{H}$ NMR spectra of complexes 16-19 were recorded in an acetone- $d_{6} / \mathrm{D}_{2} \mathrm{O}$ mixture (2:1). The signals attributable to the $\mathrm{NMe}_{2}$ and benzylic protons of the cis-coordinated dmba ligands are similar to those observed for complexes $\mathbf{3 a}$ or $\mathbf{3 b}$, respectively (see Table 6). The pyridyl resonances appear as a set of doublets for $\mathbf{1 6}$ and two broad singlets for $\mathbf{1 7}$. For $\mathbf{1 8}$, these resonances appear as two doublets, a triplet, and a singlet, and for $\mathbf{1 9}$, four broad signals were observed. With respect to the corresponding free pyridine complexes, a downfield shift for the resonances attributable to the $\alpha$ protons of the pyridyl groups was observed for platinum complexes 16 and $\mathbf{1 8}(\Delta \delta$ $(\mathrm{H})=0.32-0.43 \mathrm{ppm})$, confirming coordination of the pyridyl groups to the platinum centers. ${ }^{21 b, d}$ The $\alpha$ proton

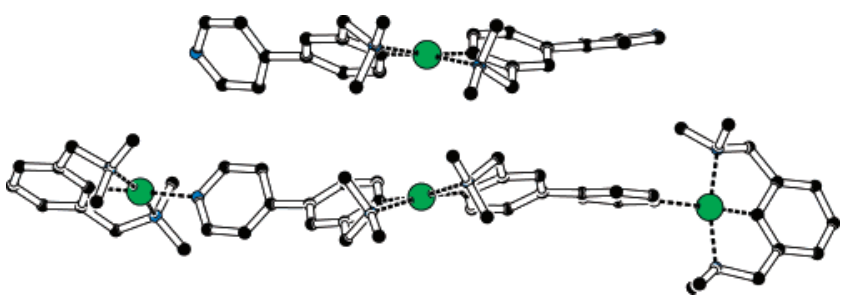

Figure 4. PLUTON plot of the side views of $\mathbf{3 a}$ (top) and the dication of 16 (bottom).

pyridyl resonances of palladium complexes $\mathbf{1 7}$ and $\mathbf{1 9}$ showed a similar downfield shift, although considerably smaller $(\Delta \delta(\mathrm{H})=0.22-0.26 \mathrm{ppm})$, while the signals are more broadened. This is a consequence of the weaker nitrogen-to-metal coordination bond in the case of palladium.

To determine the difference in the coordination behavior of the analogous palladium complex versus the cationic platinum pincer complex, $\mathbf{1 1}$ and $\mathbf{1 2}$ were reacted in a 1:1 ratio with 3a, forming [cis-Pt(4-\{p-pyridyl $\left.\} \mathrm{dmba})_{2}[\mathrm{Pt}(\mathrm{NCN})]\right]-$ $\left(\mathrm{NO}_{3}\right)$ (20) and [cis-Pt $\left.(4-\{p \text {-pyridyl }\} \mathrm{dmba})_{2}[\mathrm{Pd}(\mathrm{NCN})]\right]-$ $\left(\mathrm{NO}_{3}\right)$ (21), respectively. In the case of the mono-NCNplatinum complex 20, ${ }^{1} \mathrm{H}$ NMR spectroscopy revealed two sets of sharp resonances for coordinated and noncoordinated pyridyl groups in a 1:1 ratio, indicating that the exchange reaction for the coordination complex in solution is slow or nonexistent on the NMR time scale. In contrast, the monoNCN-palladium complex 21 undergoes a fast exchange reaction, showing in its ${ }^{1} \mathrm{H}$ NMR spectrum an average 
resonance pattern for the $\alpha$ and $\beta$ protons from the coordinated and noncoordinated pyridyl groups at 7.80 and $8.74 \mathrm{ppm}$. This is an upfield shift $\Delta \delta(\mathrm{H})$ amounting to 0.07 ppm, as compared to $\mathbf{1 7} .^{21 \mathrm{c}}$

In addition, variable-temperature ${ }^{1} \mathrm{H}$ NMR experiments were carried out to further monitor the coordination behavior of 16-19. For the platinum complexes 16 and 18, no significant spectral differences were observed in a temperature range of -35 to $+55^{\circ} \mathrm{C}$. A small upfield shift of $\Delta \delta$ (H) $\sim 0.03 \mathrm{ppm}$ of the $\alpha$ protons of the pyridyl groups was noted upon warming of solutions of $\mathbf{1 6}$ and $\mathbf{1 8}$ in a mixture of acetone- $d_{6}$ and $\mathrm{D}_{2} \mathrm{O}(2: 1, \mathrm{v} / \mathrm{v})$ from -35 to $+55^{\circ} \mathrm{C}$. At $+55{ }^{\circ} \mathrm{C}$, the resonances still appear as sharp signals, indicating that exchange reactions are still slow at this temperature. It can, therefore, be concluded that the $\mathrm{Pt}-\mathrm{N}$ bond strength is larger than that of the $\mathrm{Pd}-\mathrm{N}$ bond. The palladium complexes $\mathbf{1 7}$ and $\mathbf{1 9}$ showed a significantly larger shift of $0.18-0.28 \mathrm{ppm}$ upon increasing the temperature from -35 to $+55^{\circ} \mathrm{C}$, while sharpening of these signals was also observed. Variable-temperature ${ }^{1} \mathrm{H}$ NMR experiments for the mono-NCN-platinum complex $\mathbf{2 0}$ showed in the temperature range of -35 to $+55{ }^{\circ} \mathrm{C}$ no significant chemical shift changes. However, the ${ }^{1} \mathrm{H}$ NMR spectrum of 21 at $-35{ }^{\circ} \mathrm{C}$ showed four resonances for coordinated and noncoordinated pyridyl groups in a $1: 1$ ratio at $8.90,8.44,7.97$, and 7.59 ppm, identical with the chemical shifts of 20 at $25^{\circ} \mathrm{C}$. This indicates that below $-35^{\circ} \mathrm{C}$ the exchange reaction reaches the slow exchange limit. As was observed for complexes $\mathbf{3}$, the assembled complexes 16-19 have a $C_{2 v}$ symmetry in solution in the complete measured temperature region. As expected, the pincer metal groups do not hinder twisting of the arene rings around the platinum center.

\section{Conclusions}

Two neutral homoleptic, pyridyl-functionalized bis $(C, N$ ortho-chelated aminoaryl) platinum(II) corner complexes were synthesized via an unconventional procedure, which implies first the synthesis of a homoleptic bromide-functionalized precursor platinum(II) complex and second the subsequent functionalization with pyridyl groups in SuzukiMiyaura $\mathrm{C}-\mathrm{C}$ coupling reactions. The pyridyl-functionalized corner complexes were active as 2-fold Lewis bases in coordination reactions with the cations of NCN-platinum(II) and palladium(II) nitrate complexes, resulting in the formation of dicationic trimetallic coordination complexes. The pyridyl-functionalized corner complexes show excellent potential to be used as Lewis base functionalized corner complexes for the synthesis of larger self-assembled molecular architectures. Further studies in this direction are currently under investigation. With IR and Raman analysis, it was found that for the $\mathrm{NCN}-\mathrm{Pt}^{\mathrm{II}}$ and $-\mathrm{Pd}^{\mathrm{II}}$ complexes $\mathbf{1 1}$ and 12 both nitrate anions and water molecules are coordinated to the metal centers. In aqueous solutions, however, only aqua complexes $\left[\mathrm{M}(\mathrm{NCN})\left(\mathrm{OH}_{2}\right)\right]\left(\mathrm{NO}_{3}\right)$ were observed, indicating a complete shift in the equilibrium toward waterto-metal coordination.

Acknowledgment. M.L. and A.L.S. kindly acknowledge the Netherlands Organization for Scientific Research (NWO) for financial support.

Supporting Information Available: CIF data for 3a, 11a, 11a $\mathrm{H}_{2} \mathrm{O}$, and 16. This material is available free of charge via the Internet at http://pubs.acs.org.

IC050697V 